\title{
Advances in closed-loop deep brain stimulation devices
}

\author{
Mahboubeh Parastarfeizabadi ${ }^{*}$ and Abbas Z. Kouzani
}

\begin{abstract}
Background: Millions of patients around the world are affected by neurological and psychiatric disorders. Deep brain stimulation (DBS) is a device-based therapy that could have fewer side-effects and higher efficiencies in drug-resistant patients compared to other therapeutic options such as pharmacological approaches. Thus far, several efforts have been made to incorporate a feedback loop into DBS devices to make them operate in a closed-loop manner.

Methods: This paper presents a comprehensive investigation into the existing research-based and commercial closed-loop DBS devices. It describes a brief history of closed-loop DBS techniques, biomarkers and algorithms used for closing the feedback loop, components of the current research-based and commercial closed-loop DBS devices, and advancements and challenges in this field of research. This review also includes a comparison of the closed-loop DBS devices and provides the future directions of this area of research.

Results: Although we are in the early stages of the closed-loop DBS approach, there have been fruitful efforts in design and development of closed-loop DBS devices. To date, only one commercial closed-loop DBS device has been manufactured. However, this system does not have an intelligent and patient dependent control algorithm. A closed-loop DBS device requires a control algorithm to learn and optimize the stimulation parameters according to the brain clinical state.

Conclusions: The promising clinical effects of open-loop DBS have been demonstrated, indicating DBS as a pioneer technology and treatment option to serve neurological patients. However, like other commercial devices, DBS needs to be automated and modernized.
\end{abstract}

Keywords: Deep rain simulation, Closed-loop control, Biomarker, Sensor, Signal conditioning, Stimulator

\section{Background}

Deep Brain Stimulation (DBS) can be classified into open-loop (also known as conventional) and closed-loop (also known as adaptive) paradigms. Closed-loop DBS employs a sensor to record a signal linked to symptoms while open-loop DBS does not use a sensor for recording the brain condition; therefore, stimulation parameters including duration, amplitude, and frequency of the pulse train remain constant in open-loop DBS regardless of fluctuations in the disease state. The recorded signal is known as a biomarker and can have varying nature, e.g. bioelectric, physiologic, biochemical, etc. In the open-loop DBS, a specialist tracks the patient's clinical state and manually programs the device in a trial-and-

\footnotetext{
*Correspondence: mparasta@deakin.edu.au

School of Engineering, Deakin University, Waurn Ponds, VIC 3216, Australia
}

error based manner. Adjustments of stimulation parameters are not conducted in real-time based on the ongoing neurophysiological variations in the brain; therefore, adverse effects on the patient may be induced due to the brain overstimulation. On the other hand, in the closed-loop DBS, the stimulation pulses are delivered when the brain is in an abnormal state, or they are automatically and dynamically adjusted based on the variations in the recorded signal over the time. Figure 1 compares open-loop and closed-loop DBS and illustrates how they act in different brain states.

Although the conventional DBS is a successful therapy, the closed-loop DBS is potentially capable of further and more efficient improvements in neurological diseases. A systematic review of the clinical literature by Hamani et al. [1] stated that adjusting the stimulation 

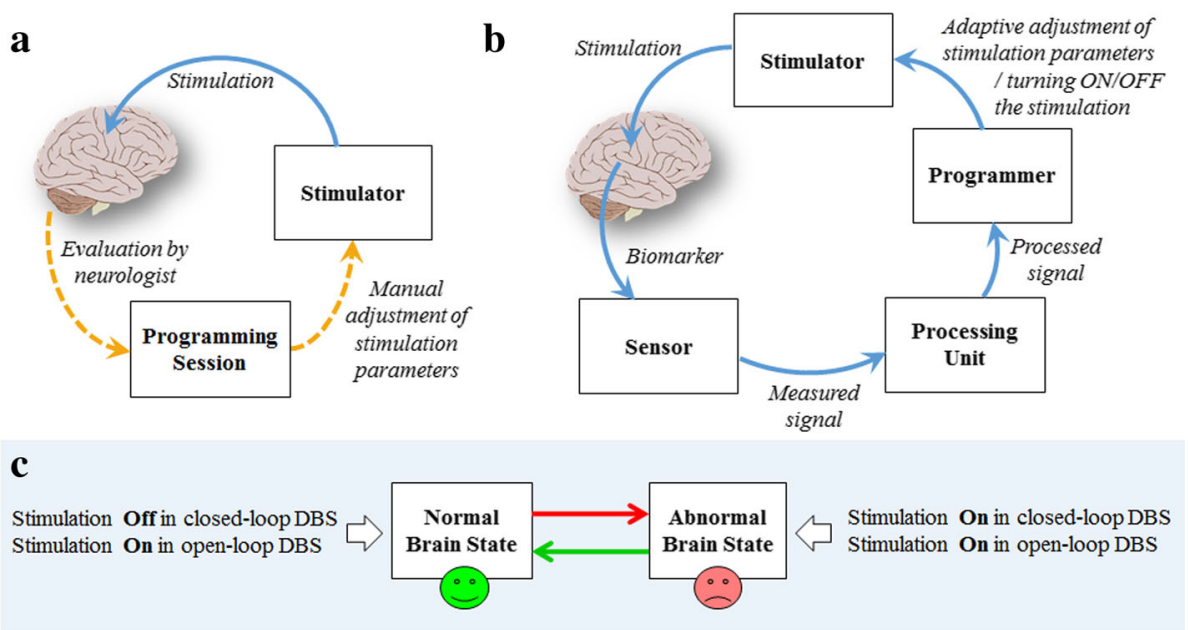

Fig. 1 Overview of open-loop DBS (a) versus closed-loop DBS (b). In open-loop DBS, a neurologist manually adjusts the stimulation parameters every 3-12 months after DBS implantation. On the other hand, in closed-loop DBS, programming of the stimulation parameters is performed automatically based on the measured biomarker. c Demonstration of two different brain states and the action of open-loop and closed-loop DBS. When the brain enters a specific state, it remains in that state for a short or long time. Closed-loop DBS gets deactivated when the brain enters the normal state. Open-loop DBS continues the stimulation regardless of the brain state

parameters of DBS devices could reduce or abolish adverse effects reported in 142 (19\%) of 737 Parkinson's disease (PD) patients treated with subthalamic nucleus (STN) DBS. In addition, Rosin et al. [2] demonstrated the superior function of closed-loop DBS, which automatically adjusts the stimulation parameters, to alleviate PD symptoms. Moreover, Little et al. [3] indicated that motor scores in eight PD patients improved by $50 \%$ (blinded) and 66\% (unblinded) during closed-loop DBS, which were $27 \%(p=0.005)$ to $29 \%(p=0.03)$ higher than that of open-loop DBS. Besides these therapeutic benefits, they reported 56\% reduction in stimulation time, as well as a decrease in the energy requirement of the closed-loop DBS compared to open-loop DBS. Therefore, patients may also benefit from fewer surgeries for replacement of the neurostimulator battery as a result of less power consumption in non-continuous stimulations [3]. Little et al. [3] and Wu et al. [4] reported that in order to obtain similar results from open-loop and closed-loop DBS, $44 \%$ less electrical stimulation is required using closed-loop DBS, which means higher efficiency, fewer surgery numbers, lower power consumption, and longer battery lifespan.

Although DBS is a successful therapy, its operation mechanism is mainly uncertain. Hess et al. [5] explained how the temporal pattern of stimulations might have key information for clarification of the DBS mechanism. A recent short review [6] on the physiological mechanism of DBS suggests the "disruption hypothesis" in which abnormal information is prevented from flowing into the stimulation site as a result of DBS dissociation effect on input and output signals. However, it is still under debate and remains to be confirmed by more preclinical research. Another review by Herrington et al. [7] accounts several non-exclusive mechanisms for DBS that depend on the condition being treated and the stimulation target. Despite the existence of different theories on the DBS mechanism, there are still questions in regard to the closed-loop DBS. Does adaptive control of DBS alter the DBS mechanism? If yes, how does it alter the DBS mechanism? These questions deserve consideration in the future experimental studies.

This paper presents a comprehensive review of portable closed-loop DBS devices. While there exists a number of excellent reviews on closed-loop DBS systems [8-16], this work differs from the existing works as described in the following. Among the published reviews, ref. [8] mainly highlights the applications of closed-loop DBS in the rehabilitation of movement disorders. Ref. [12] mainly describes the benefits of closed-loop DBS which using local field potentials (LFPs) as the feedback biomarker. Ref. [13] mainly reviews DBS (both open-loop and closed-loop) in terms of neurological aspects and clinical benefits. Ref. [9] indicates the available biomarkers for closing the feedback loop, and gives control strategies for manipulating measured signals relating to PD patient clinical state. Ref. [10] concentrates on emerging techniques in DBS including new electrode design, new stimulation patterns, and novel targeting techniques. Ref [16] has mainly focused on selection of biomarker and its benefits and problems. Ref. [14] introduces adaptive DBS, and outlines some technological advances in DBS including stimulation type and patterns, energy harvesting, and methods for increasing life quality of patients. Similarly, ref. [15] reviews some technological 
advancement such as surgical targeting, DBS parameters programming, and electrode design. On the other hand, ref. [11] highlights a range of issues associated with closedloop DBS including biomarker sensing and processing, DBS parameters programming, control algorithm, wireless telemetry, and device size and power consumption.

This paper, on the other hand, provides a comprehensive review of closed-loop DBS devices, and covers a wider range of issues and advancements associated with such devices including: (i) biomarker selection, (ii) DBS parameters programming, (iii) stimulation type and pattern, (iv) control algorithms, (v) concurrent stimulation and recording, (vi) portability, (vii) battery-less technique, (viii) user-friendly interface, and $(\mathrm{x})$ remote monitoring and wireless telemetry. The paper combines the key features of the current reviews going beyond devices that are used for specific disorders or biomarkers. It covers closed-loop DBS devices reported in the latest research publications not included in the existing reviews. The paper gives a brief history of closed-loop DBS. Next, it discusses different biomarkers for closing the feedback loop. Then, it reviews the algorithms developed for controlling stimulation parameters. After that, it highlights the current challenges and limitations for implementing closed-loop DBS. Also, it reviews the technological developments in closed-loop DBS. Then, it describes commercial closed-loop DBS systems. After that, it compares researchbased closed-loop DBS devices highlighting future design expectations, and giving future directions and recommendations on closed-loop DBS devices.

\section{Brief history of closed-loop DBS}

The review of scientific literature reveals that the initial use of closed-loop against open-loop DBS goes back to early 2000 [17] when an ultra-short-term closed-loop neurostimulator device was introduced being capable of stimulation by detection of seizures. This initial pilot study led to optimistic findings which were confirmed by other studies during 2002-2005. These studies $[18,19]$ concentrated on an external responsive neurostimulator (RNS) system capable of detecting seizures, delivering stimulations in a semi-closedloop manner, and storing electrocorticogram (ECoG) potentials. These studies had promising consequences on seizure activity of 11 out of 27 patients. However, due to the low specificity of seizure detection of the RNS [20], it was not entirely considered as an adaptive DBS triggered by seizures [21].

Following the use of a semi-closed-loop DBS device on epileptic patients, the idea of dynamically controlling DBS became a target for many researchers. However, implementation of closed-loop DBS was postponed due to the difficulties associated with technical and computational aspects, and the reliability of selected biomarkers. Selection of biomarkers and control algorithm for epilepsy could be less complicated than that for other neurological disorders (because of a distinguishable shape of the seizures activities compared to the non-seizure neural signal). Thus, lack of reliable biomarkers for most of neurological disorders postponed the development of a closed-loop DBS device for such disorders. Later, various investigations were initiated to address the problems associated with the use of closed-loop DBS for several neurological disorders. These attempts have been performed towards developments of closed-loop DBS devices, which are discussed in detail in the later sections of this review.

\section{Biomarkers for closing of the feedback loop}

It is crucial for a closed-loop DBS device to take advantage of a feedback signal in the control loop to eliminate the problems associated with open-loop adjustments of stimulation parameters. Open-loop adjustment of stimulation parameters is not an efficient procedure. In the past decade, various physiological signals have been used as a feedback in the closed-loop DBS systems. Action potentials (APs) [2, 22], ECoGs [23, 24], LFPs [3, 12, 25-28], and electroencephalogram (EEGs) [29] are examples of electrophysiological biomarkers considered in the feedback loop of adaptive DBS systems (see Fig. 2 for details on the layer of extraction). Aside from electrophysiological signals, some researchers have focused on creating the feedback loop using other biomarkers such as electromyogram (EMG) [30-33], and biochemical [34] signals. In addition, Hebb et al. proposed the optical and mechanical signals as other possible control signals [9]. However, these signals require further assessments in terms of their practicality. Each biomarker comprises merits and demerits concerning invasiveness, signal content, and resolution, as well as suitability for a disorder type [9] (see Table 1).

The cortical EEG in PD patients have shown relevance to abnormal basal ganglia circuit functions [29]. In addition, high thalamocortical theta coherence has been verified in PD patients [35]. These features indicate that the cortical EEG signal could be used as a relevant biomarker to the PD symptoms, especially because of its non-invasive nature. However, it suffers from low amplitude (maximum 10-20 $\mu \mathrm{v}$ [36]), low spatial resolution ( 3-9 cm [37, 38]), high noise and artifacts sensitivity (e.g. to eye movements [39]), as well as lack of high-frequency components (typically between $0.5-40 \mathrm{~Hz}$ [36]). These limitations mainly arise from longer distance of the sensing electrode from the neuronal potential sources. In addition to the stated limitations, the patient's freedom of movement is restricted through the non-implantable electrodes. Therefore, it causes discomfort to the patient due to the attachment of electrodes to the head. 


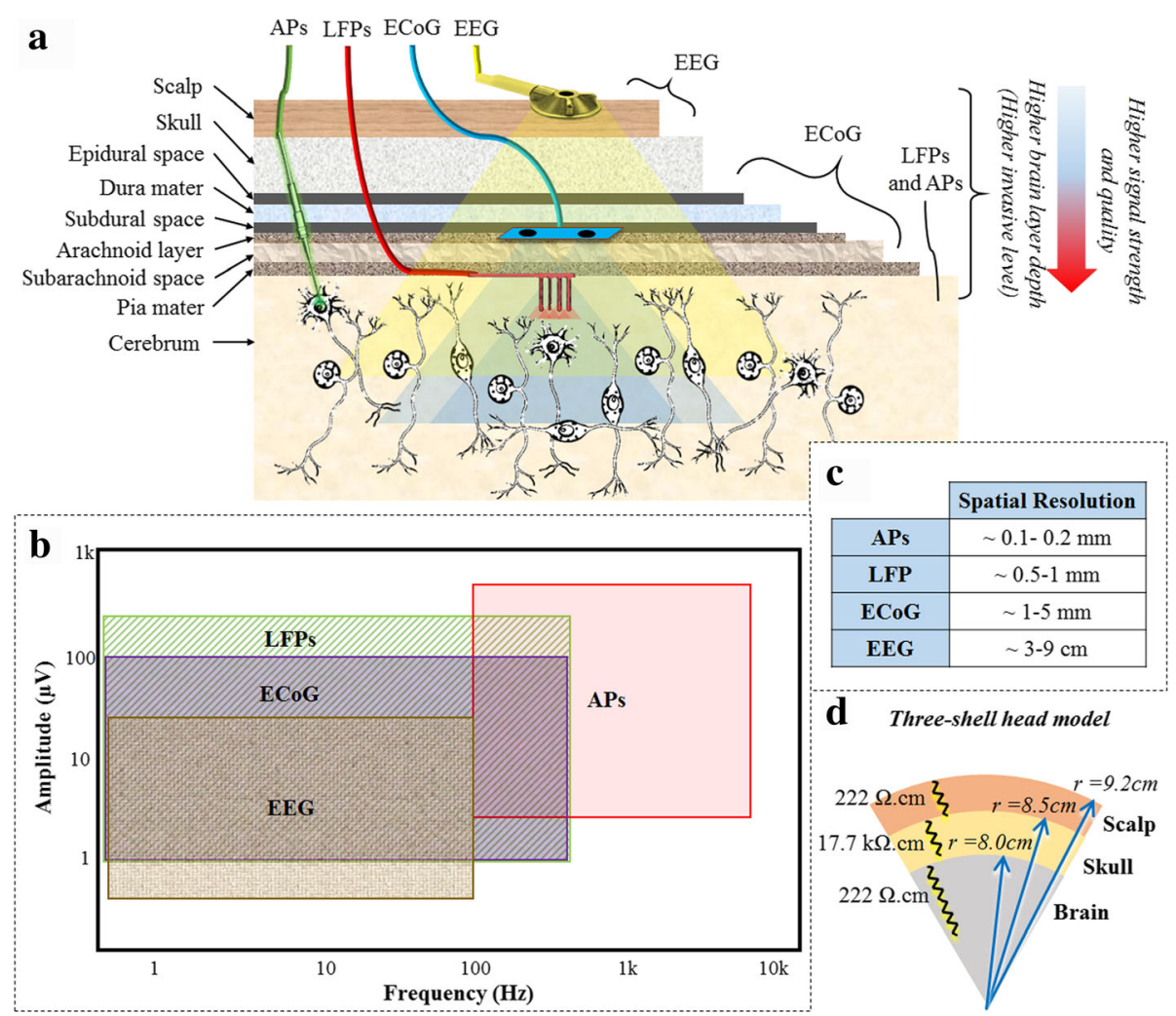

Fig. 2 a A schematic representing different brain layers and measurable electrophysiological signals. Recording from higher depths results in potentials with higher strength and quality. The higher the distance of electrode from the potential source means a larger impedance. Therefore, proportional to the distance, the potentials are attenuated and high-frequency components are rejected due to the low-pass filtering behavior of the brain layers [159, 160]. In addition, recording from an electrode with smaller contact area enables measuring potentials from fewer neurons [161]. b Amplitude vs frequency characteristics of the human brain potentials of interest. c The spatial resolution of electrophysiological signals. $\mathbf{d}$ Three-shell head model. Different layers of the head, particularly the skull with a large resistivity, induce a distorting effect on the potentials that reach the scalp surface

ECoG as another biomarker has been used by Afshar et al. study [23]. They proposed an ECoG-based brainmachine interface system and presented a correlation of ECoG with the disease state. In comparison with EEG, ECoG has higher signal quality in both amplitude and frequency ranges (maximum 50-100 $\mu \mathrm{v}$ with frequencies between $0.5-500 \mathrm{~Hz}$ [36]). This feature has made ECoG a signal with higher popularity in neuroscience research, especially for brain-computer interface (BCI) applications. However, selecting ECoG as a biomarker requires placement of subdural electrodes in the brain epidural or subdural spaces, which are still away from the neuronal stimulation site. Thus, the concerns on the spatial resolution of ECoG signals may still exist. For instance, the spatial resolution deteriorates significantly from $1.25 \mathrm{~mm}$ to $1.4 \mathrm{~mm}$ by placing the electrode on the epidural instead of subdural space [40, 41]. Moreover, the difference in the recording and stimulation electrodes may yield additional costs and longer implantation and anesthesia duration that may impose further brain injuries.

In contrast, APs, because of their high spatial resolution (maximum $0.2 \mathrm{~mm}$ [38]), can be a good candidate as a biomarker for controlling adaptive DBS devices. Typical APs recorded extracellularly have an amplitude of up to $500 \mu \mathrm{V}(100 \mathrm{mV}$ intracellularly [42]) with frequencies between $100 \mathrm{~Hz}-7 \mathrm{kHz}$ [43]. However, continuous recording of APs can cause death of neurons (if intracellular). Moreover, there is a need for recalibration processes to keep the stability of feedback signal [44]. Thus, these limitations prevent the use of APs as a biomarker for long-term stimulation. Furthermore, other biomarkers such as biochemical potentials need stabilization of the carbon fiber microelectrode during recording [45]. Furthermore, applying other biomarkers such as EMG seem to have similar limitations as EEG. Moreover, EMG is linked to only a limited number of diseases including PD and essential tremor [30-33].

On the other hand, LFPs are the most used feedback signal in closed-loop DBS [3, 12, 26]. LFPs, also known as intracranial EEG [46], are potentials generated in the extracellular space by propagation of APs through axons. These local potentials reflect neuronal processes occurring within a local region around electrode in the neuronal extracellular space [47]. Priori et al. review [12] 


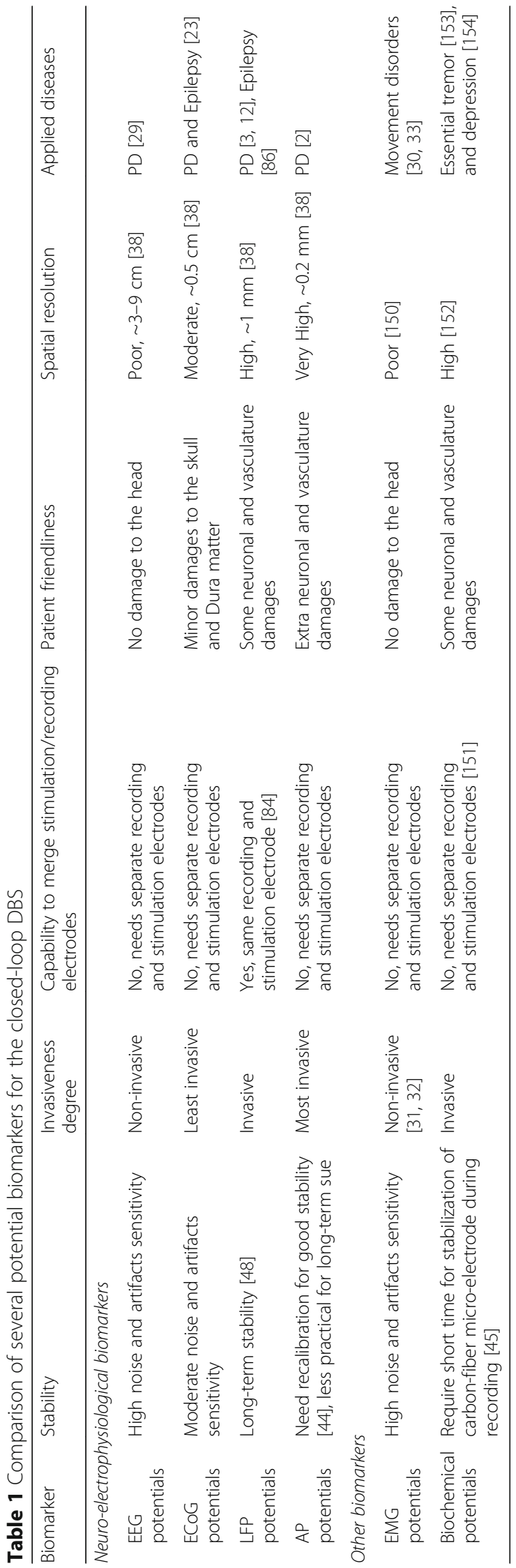


demonstrated the suitability of LFPs as the feedback signal in closed-loop DBS devices for PD patients. One key advantage is that LFPs can be directly recorded from the stimulation electrodes. Another advantage of LFPs is the long-term stability achieved at the electrode-tissue interface [48]. LFPs generally have amplitudes of up to $200 \mu \mathrm{V}$ with energies below $500 \mathrm{~Hz}$ [49]. Compared to APs, LFPs have a reasonable spatial resolution, typically around $1 \mathrm{~mm}$ [38]. As discussed in a review by Deeb et al. [50], thus far several neurological disorders have been investigated with closed-loop DBS systems controlled by LFPs. While PD and epilepsy have been mainly focused on, other disorders including Tourette syndrome, major depression, and tremor have been also tried recently.

Generally, the selection of a proper biomarker depends on several factors. It is usually chosen with respect to the disease type and the degree of relevance to the disease symptoms. Apart from being linked to a symptom, it is vital for a biomarker to be recorded with a high signal to noise ratio, and most importantly be stable and unaffected by external artifacts such as movement, talking, and thinking [11].

\section{Control of feedback and stimulation parameters}

Automatic and non-subjective optimization of closedloop stimulation parameters can enhance the patients' therapeutic benefits while minimizing the side effects. Although the neurologists reprogram the open-loop DBS to enhance the therapeutic results, the procedure is not optimal and would not deliver the best therapeutic effects [2]. Simultaneous and automatic control of stimulation parameters could improve the effectiveness of therapy. A schematic of the closed-loop DBS programming process is illustrated in Fig. 3.

Several neurological diseases are caused by synchronized populations of oscillatory neurons. In some patients, open-loop DBS may not yield desynchronization or its therapeutic effect may decline over time [51]. In particular, the brain reaction to a constant set of stimulation parameters may change gradually over time due to the external (environmental) or internal (disease progress, and behavioral) factors $[52,53]$. This procedure is called neural plasticity which is the ability of the nervous system to adopt a new functional or structural state in response to extrinsic and intrinsic factors [54].

During the past decades, several model-based closedloop stimulation methods have been developed in order to restore desynchronized dynamics in networks of oscillatory neurons. These methods include single-site linear $[55,56]$, multi-site linear [57, 58], and non-linear [59-62] delayed feedback stimulation techniques, which intrinsically operate in a closed-loop or demand-controlled manners. Another model-based method for desynchronization of oscillatory neurons was proposed by Tukhlina et al.
[63] which is an implementation of a phase shifter. In this method, suppression of neural synchrony is realized by organizing an interaction between the ensemble and a passive oscillator [63]. In recent published works [64, 65], Popovych et al. combined the closed-loop DBS approach with desynchronizing stimulation protocols. They extended linear and non-linear delayed feedback stimulation methods to a pulsatile closed-loop DBS, and showed effective and robust desynchronization of STN-GPe model neurons. In addition, they showed that the presence of an interphase gap between the recharging phases of the charge-balanced biphasic pulses can significantly improve the stimulation-induced desynchronization in a closedloop DBS. Here, the amplitude of the stimulation pulses was adjusted by the LFP feedback signal based on linear or nonlinear delayed feedback rules.

In addition to the above presented works, there exist many other interesting reports on development of an optimized controller for programming of stimulation parameters. Santaniello et al. [66] developed a modelbased controller through simulation of intrinsically active neurons in the Vim thalamus and getting LFPs from the neurons. This controller works based on a recursive autoregressive model and adjusts the stimulation amplitude automatically based on the feedback signal recorded from the stimulation electrodes. Pyragas et al. [67] presented a demand-controlled method for suppression of neural synchrony using a proportionalintegral-derivative (PID) feedback, which enables robust restoration of desynchronized states. Similarly, Gorzelic et al. [68] and Dunn et al. [69] suggested model-based algorithms that operate based on PID feedback. Their suggested algorithms enable optimization of stimulation parameters in the closed-loop DBS. In addition, an adaptive feedback input-output linearization algorithm for closedloop control of PD has been proposed by Su et al. [70]. This algorithm adjusts the input signal based on the estimation of parameters from the feedback signal. Rhew et al. [25] designed a log-based adaptive DBS device that utilizes lowpass-filtered LFPs in a proportional-integral (PI) controller to define the optimum stimulation amplitudes. This system works based on biomarker energy and benefits from a robust feedback controller because of removing high-frequency artifacts from the controller input. Another interesting controller has been designed that operates based on variations in the neurochemical release [34]. The integration of recording and stimulation modules is facilitated via fast-scan cyclic voltammetry (FSCV). This system records artifact-free evoked neurochemical alterations to wirelessly control the stimulation parameters [34]. Another neurochemical controller is proposed by Grahn et al. [71] in which evoked dopamine changes are recorded to adapt the stimulation parameters in a rodent model. 


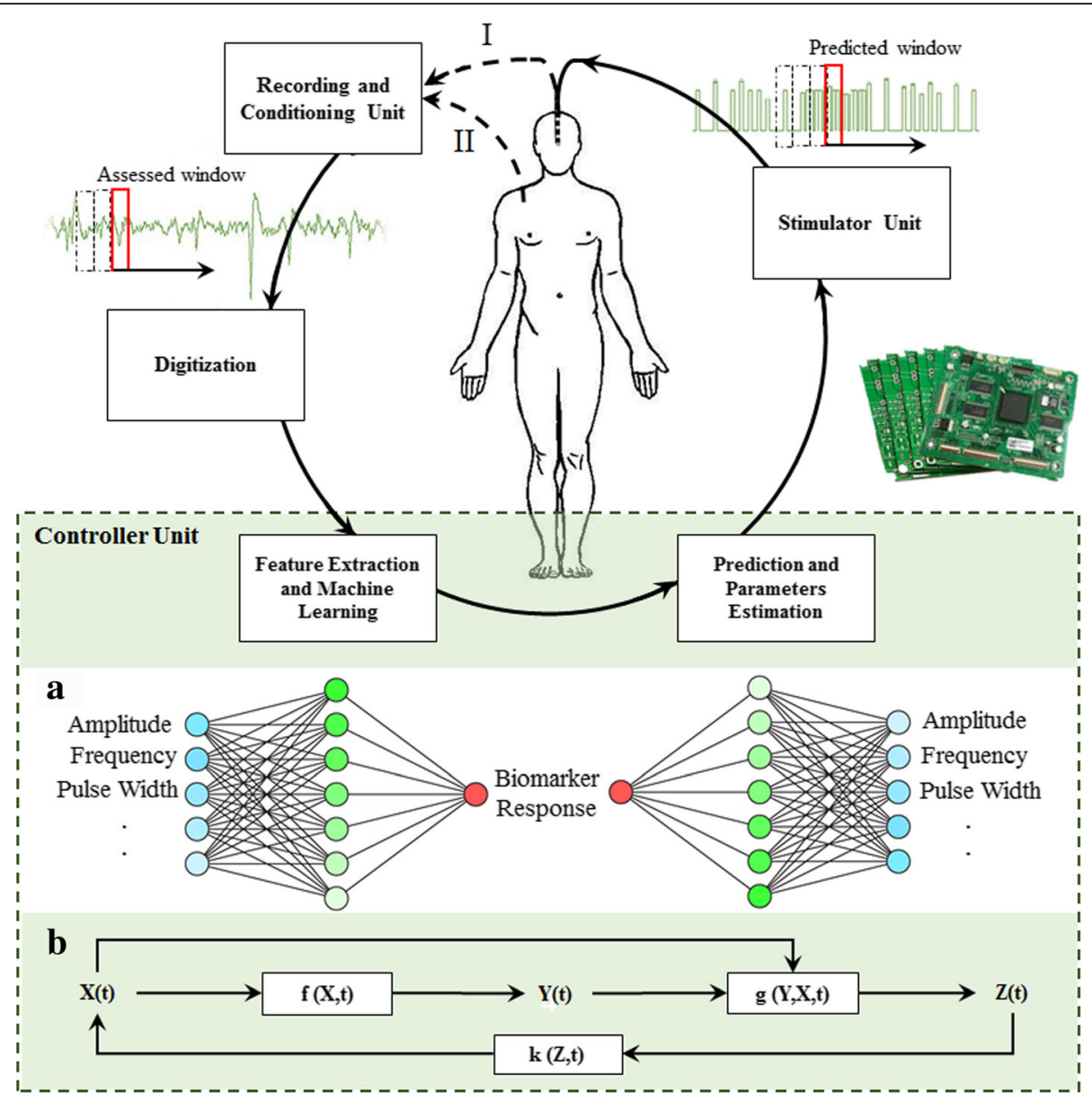

Fig. 3 The process of real-time closed-loop DBS programming. The recording unit records the biomarker signal via an inserted electrode inside (I) or outside (II) of the brain based on the biomarker type. After signal conditioning (amplification and filtration), the biomarker signal is digitized and then sent to the controller unit. Then, through a computational model (A), the biomarker signal is evaluated from different aspects (e.g. amplitude, frequency, and pulse-width, etc.) to define the response signal, which is then employed to predict optimized stimulation parameters. The bottom model (B) has been adopted from [105] and then modified. It represents the structure of controller where $X(t), Y(t)$, and $Z(t)$ are the input vector, neural circuit states, and biomarker response, respectively. The mapping functions from input to the neural state and from neural state to the biomarker response are demonstrated by $f(X, t)$ and $g(X, Y, t)$, respectively. The $k(Z, t)$ is the controller that evaluates the biomarker response and updates stimulation parameters. The estimated parameters are adjusted in the stimulation unit to create the stimulation pulses for applying to the stimulation electrodes. In this real-time process, a very short time-window of the recorded signal is used for prediction of the stimulation parameters. The time-window of biomarker signal is pushed forward continuously and simultaneous computations are done to predict and update the next stimulation-window

Some of these closed-loop controllers, however, only control one of the pulse parameters (usually amplitude) or just implement a simple ON-OFF control of stimulations. The on-demand systems trigger the DBS switch to act only when the stimulation is needed. It is a simplified form of closed-loop DBS capable of preventing brain over-stimulation. The idea is to set a threshold and check the biomarker continually, finding when it crosses the threshold to turn on or off the stimulations. This type of strategy to control the stimulation pulses in a closed-loop DBS is based on an "amplitude-responsive" strategy [72]. Numerous studies focus on the increment of beta frequency power $(13-35 \mathrm{~Hz})$ in PD and tremor [73-75]. Little et al. [3, 76, 77] designed an on-demand adaptive DBS that digitally filters the biomarker around the beta frequency $(22 \mathrm{~Hz})$, generating a smoothed beta amplitude. Then, they utilize it as an input to the controller that regularly checks the beta oscillatory amplitude with respect to a personalized threshold (different in each patient). Instead of providing an on-off strategy, Rosa et al. [78] presented an algorithm that continuously and linearly modifies the stimulation parameters each second according to the changes in the patient's LFP beta power $(13-17 \mathrm{~Hz})$.

One of the concerns about the use of the beta frequency band as a feedback biomarker is that it might be affected and suppressed by movement [79]. This issue is not an important problem in the experimental studies 
when the patient is motionless. However, in a beta-based closed-loop DBS device implanted in the patient's brain, the patient's movements can supress the beta amplitude which may degrade the closed-loop performance. Another concern relates to the accuracy of the promising outcomes of beta-based studies due to the fact that they only test the patients in a temporary time period (after electrode implantation and before surgery to implant the pulse generator, when the leads are accessible). This period, known as post-operative "stun effect", is believed to causes unrealistic outcomes as a result of temporary alleviation of PD symptoms even without stimulation [80, 81]. However, a recent published paper [82] presents a proof of principle to the chronic application of adaptive DBS, and confirms the accuracy of the closed-loop DBS in the temporary period. Piña-Fuentes et al. [82] presented the first case of closed-loop DBS in a PD patient with chronic STN-DBS treatment, and concluded that closed-loop DBS can be applied in the chronically implanted DBS phase, and is at least as effective as open-loop DBS when objectively assessed [82].

Beyond the "amplitude-responsive" strategy for controlling the stimulation pulses in a closed-loop DBS, there is another strategy termed "phase-responsive". In a phase responsive closed loop DBS, the stimulations are directed by the phase (timing) of the biomarker signal [72]. The phaseresponsive closed-loop DBS is currently being developed for treatment of tremor, aiming to selectively stimulate at the phases that attenuate the tremor amplitude through an accelerometer attached to the tremulous hand $[72,83]$. The results of this control strategy show a significant tremor relief in essential tremor patients [83]. Thus far, the phaseresponsive closed-loop DBS has not been applied for longer than $30 \mathrm{~s}$ [72].

\section{Technological advancements towards closed-loop DBS devices}

In this section, the existing closed-loop DBS systems both from hardware (circuit design) and software (control algorithm and programming) perspectives are reviewed.

\section{Stimulation artifact suppression}

The stimulation current pulses can cause interference with reading of biomarkers of interest. This interference makes it difficult to accurately record and process biomarkers because of the large difference in the amplitude of the stimulation pulses and the measured signal. For instance, the magnitude of LFPs is roughly five to six fold (100-120 dB) smaller than that of the stimulation pulses [84]. This difference yields a combination of strong stimulation artifact (in volts range) with a weak neural signal (in $\mu \mathrm{V}$ range), which saturates the amplifiers used in the recorder.
To alleviate this problem, Rossi et al. [84] designed "FilterDBS" which is an artifact-free recording system for acquisition of LFPs from the DBS lead positioned in the STN. The $130 \mathrm{~Hz}$ stimulation artifact and the higher harmonics were separated in the frequency domain using a bandpass filter $(2-40 \mathrm{~Hz})$. This system benefits from an overall gain of $100 \mathrm{~dB}$ with $130 \mathrm{~dB}$ common mode rejection ratio (CMRR). However, the device requires a $\pm 15 \mathrm{~V}$ supply to operate. Post-filtering is another method to remove the stimulation artifact [85], in which the template of the stimulation signal is subtracted from the recorded signal to produce an artifact-free biomarker. However, this method degrades the signal quality which is not desirable. Moreover, it may not operate correctly in the closed-loop DBS where the stimulation rate fluctuates.

Stanslaski et al. [86] designed an implantable, chronic, adaptive DBS device that benefits from an LFP/ECoG sensor. This device was validated successfully in an ovine model of epilepsy. The hippocampus seizure activity was detected and measured during and after stimulation. The separation of the biomarker from the stimulation artifact was conducted through a support vector machine classification algorithm by processing of the spectral fluctuations. The suggested device fits in a $39 \mathrm{~cm}^{3}$ space, employing front-end filtering that guarantees the op-amp input to be within its normal operation area [86]. In another work, Zbrzeski et al. [87] introduced an integrated neural amplifier to reduce artifacts in the recording of LFPs and spikes. The proposed neural amplifier occupies a space of $0.15 \mathrm{~mm}^{2}$ and consumes $6.73 \mu \mathrm{W}$ of power. Test-bench validation of the amplifier showed a mid-band gain of $20 \mathrm{~dB}$ with a low input-referred noise of $4 \mu \mathrm{V}_{\text {rms }}$. However, despite the advances in the simultaneous recording and stimulation, the issue of stimulation artifact in the recorded signal has not been fully addressed yet in the existing DBS systems.

\section{Stimulation challenges \\ A. Stimulation pattern}

The pattern of stimulation is a major concern for both open-loop and closed-loop DBS devices. Figure 4 shows the differences between monophasic (a fully positive (cathodic) or a fully negative (anodic) pulse) versus biphasic (a pulse having both anodic and cathodic sides) stimulations. Whilst monophasic pulses are chargeimbalanced, biphasic pulses can be charge-imbalanced or charge-balanced. Being imbalanced in terms of charge yields formation of undesirable chemical reactions at the electrode contact surface over time [88]. Therefore, monophasic and charge-imbalanced biphasic pulses can be dangerous for the brain tissue due to their non-zero 


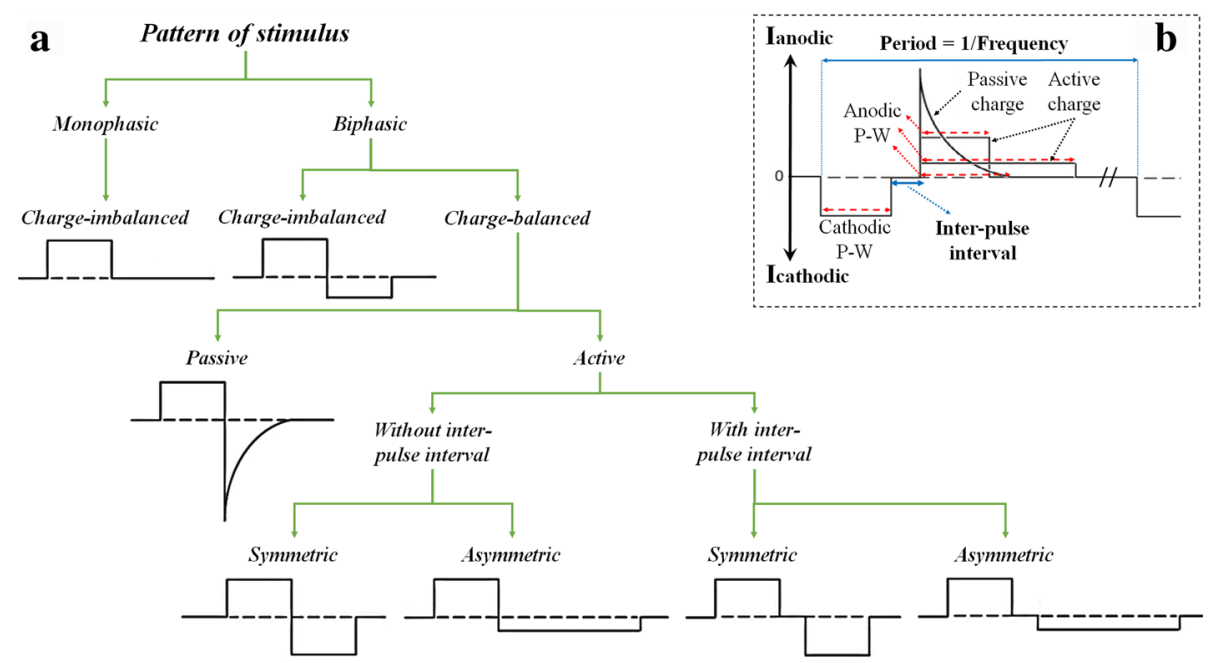

Fig. 4 Categorization of different stimulation patterns. For additional details regarding the pros and cons of each waveform refer to [89, 162]

net charge that can create a lesion. Charge-balanced pulses can be obtained through passive or active charge balancing schemes. In the active methods, the second pulse is truly built using a second current source or through a further complex circuitry with one current source. However, in the passive charge-balancing scheme, a passive components or shortening of the electrodes to the ground is used. Although charge-balanced biphasic pulses may prevent net charge injection into the stimulated tissue [88], the anodic phase neutralizes the anticipated stimulation effect of the cathodic phase [89]. Typically, an inter-pulse interval is applied to the biphasic pulses in order to avoid cells hyperpolarization and minimize the suppressing and altering effects of the anodic phase [89]. It is worth stating that most of the available market-based open-loop and closed-loop DBS systems use a passive charge-balancing scheme.

Ewing et al. proposed a DBS device named SaBer DBS [90]. It delivers biphasic, passive charge-balanced pulses to two independent channels. Immediately after delivering the stimulation pulses, the electrodes are grounded (capacitive coupling) to achieve the zero-net charge. Although it is programmable in frequency, pulse-width, and current amplitude, the user defines the stimulation parameters; thus, the system is not closed-loop. Moreover, lack of inter-pulse interval between the cathodic and anodic pulses may alter neuronal activities. Sooksood et al. [89] proposed a novel active approach for charge balancing via long-term offset regulation. Using this method, the voltage of the electrode is checked after each stimulation pulse to fall within a predefined range. If not, the mismatch is compensated via an adjusted offset current. The power-efficient DBS system designed by Lee et al. [91] uses the offset regulation method. They designed a power-efficient DBS system that employs closed-loop active charge balancing scheme (by utilizing a small balancing current pulse) to retain the charge within a safe window $(50 \mathrm{mV})$. It is also capable of estimating the residual time remained for balancing.

Apart from the pattern shape, a temporally irregular (i.e. variable inter-burst interval) stimulation pattern in the closed-loop DBS has caused concerns regarding the effectiveness of stimulation. On one hand, it has been observed that irregular stimulation patterns can reduce the beneficial therapeutic effects $[92,93]$. On the other hand, the closed-loop DBS appears to be irregular because of the adaptive variations. Rosin et al. [2] applied both closed-loop stimulation and random stimulation (unrelated to the ongoing activity) patterns in two PD monkeys. They observed that in spite of the irregular pattern of the closed-loop DBS, it is a successful therapeutic method. It was explained that the adaptive (symptom-related) nature of the closed-loop DBS induces the therapeutic effects regardless of its pattern's irregularity.

\section{B. Stimulation type}

Delivering method of stimulation pulses, whether to dispatch constant-current-controlled (CC) or constantvoltage-controlled $(\mathrm{CV})$ stimulation, is a matter of debate. Most of the available commercial DBS devices offer the CV stimulators due to their higher level of power efficiency [14]. In the CC stimulation, the dropout voltage across the current source wastes the power and reduces the device battery life. On the other hand, the current and the volume of the tissue activated in CV devices may alter because of the impedance variations in the brain tissue and the electrode-tissue interface [94]. Whereas the CC stimulators provide a more precise control independent of the impedance variations. Impedance fluctuations have been observed 
during the first 3 months following DBS surgery [95, 96] as well as post-implantation [26, 97, 98]. Satzer et al. [99] highlight a mean $73 \Omega$ /year reduction in impedance in most electrode contacts; thus, utilizing $\mathrm{CC}$ devices is more preferred. However, in terms of effectiveness, there is no proven evidence to give $\mathrm{CC}$ preference over $\mathrm{CV}$ stimulation [100]. Although better clinical outcomes were reported via $\mathrm{CC}$ stimulation in patients suffering from dystonia in the Lettieri et al. experiment [94], the study had some limitations as discussed in ref. [101].

In order to improve the power efficiency problem in CC stimulators, Azin et al. [102] proposed a closed-loop DBS device which controls the stimulator supply voltage adaptively through an inductive link. It is a 4-channel intracortical micro-stimulation integrated circuit (IC) $\left(10.9 \mathrm{~mm}^{2}\right.$ system on chip (SoC)) that converts recorded extracellular neural spikes to electrical stimulations for real-time delivering from one brain region to another [102]. It comprises a voltage readout channel to close the feedback loop. Moreover, it includes a direct current (DC) to DC converter that converts a single $1.5 \mathrm{~V}$ battery to $5.05 \mathrm{~V}$ to supply the micro-stimulating back-end circuit. Similarly, Hyung-Min et al. [91] designed a 4-channel wireless CC stimulator with a closed-loop supply control that utilizes a voltage readout channel. The voltage readout channel automatically controls the stimulation voltage and improves the efficiency (30\% higher than a fixed supply voltage). This prototype has been verified in vitro, occupies $2.25 \mathrm{~mm}^{2}$ with $5 \mathrm{~V}$ peak alternating current $(\mathrm{AC})$ input (rectified to a $\mathrm{DC}$ voltage between $2.5 \mathrm{~V}$ to $4.6 \mathrm{~V}$ ) at $2 \mathrm{MHz}$.

\section{Portable closed-loop DBS}

Another issue is the size and weight of the closedloop DBS device. Compared to the open-loop DBS devices, closed-loop systems have additional recording and programming circuits which increase the size and weight of the final design. Because the initial experiments are usually carried out in small laboratory animals, it is necessary that the device be miniature and lightweight, allowing stimulation during the animal life cycle (eating, playing, walking, sleeping, etc.). The issue is even more important when the device is expected to be used for long-term experiments. Providing a balance of functionality, portability, the number of input and output channels, and power requirements in the closed-loop DBS devices demand a comprehensive research.

Jongwoo et al. [103] developed a 64-channel programmable IC to be used as closed-loop DBS device for treatment of neurological disorders. This device employs eight low-noise front-end pre-amplifiers, single $200 \mathrm{kS} / \mathrm{s}$ 8-bit logarithmic pipeline analog to digital converter (ADC), and digital filters. It has been implemented in $0.18 \mu \mathrm{m}$ complementary metal-oxide-semiconductor
(CMOS) technology and benefits from $2.7 \mathrm{~mm}^{2}$ occupied space. Both LFPs and spikes are sensed and then separated through on-chip digital filters. The proposed device consumes $89 \mu \mathrm{W}$ in a normal mode and $271 \mu \mathrm{W}$ in a configuration mode from $1.8 \mathrm{~V}$ supply. In addition, Pinnell et al. [104] developed a miniature wireless closed-loop CC DBS with a total weight of $8.5 \mathrm{~g}$ to be used with laboratory animal. This system operates for more than $8 \mathrm{~h}$ and transmits LFPs to $3-5 \mathrm{~m}$ distances. Another recent effort towards the portability is the work done by Arlotti et al. [16]. They have developed an external portable closed-loop DBS for clinical experiments in freely-moving PD patients. This device analyses LFPs recorded from a single differential artifact-free channel. The external part weights $150 \mathrm{~g}$ occupying $12 \times 7 \times 2.5 \mathrm{~cm}^{3}$ in space. It can be worn externally by humans to investigate closed-loop DBS effectiveness. However, it is a large and heavy device for small laboratory animals.

\section{Battery-less closed-loop DBS}

Another hurdle in designing a closed-loop DBS is the possibility of continuous biomarker recording and real-time processing with least energy consumption. Some researchers have developed DBS devices that consume low power [105-107]. However, these devices have limitations with lack of re-chargeability and limited memory space. These weaknesses can impose potential surgery risks and extra costs on patients [108].

The invention of energy harvesting technology has increased the battery lifetime of DBS devices, reducing the frequency of surgical replacement procedures, and subsequently minimizing the extra costs. Harvesting the power from human or environmental sources (kinetic, electromagnetic, thermal and infrared radiant energies) have been discussed in a review by Hannan et al. [109]. The review suggests the inductive coupling link as a more suitable method of powering the battery-less implantable biomedical devices. Harrison et al. [42] designed a neural recording system in which power and command are transferred wirelessly (at $6.5 \mathrm{~kb} / \mathrm{s}$ ) via a $2.64 \mathrm{MHz}$ inductive link. In other studies, Hosain et al. [110-112] proposed a four-layer circular planar inverted-F antenna to harvest power for adaptive DBS implants and examined the feasibility of wireless transmission of power, control, and command signals. They then proposed RF rectifiers for electromagnetic power harvesting in a DBS implant [113]. Besides these, a system-on-chip wireless closed-loop DBS was developed by Hyo-Gyuem et al. [25, 114] which is capable of two-way wireless telemetry through RF energy harvesting. Lin et al. [115] proposed another battery-less, implantable neuro-electronic interface capable of two-way wireless telemetry. It receives power through a single coil and transmits data via the same coil. The device has been fabricated with the standard $0.18 \mu \mathrm{m}$ CMOS technology with a chip area of $7.74 \mathrm{~mm}$. 


\section{Patient-friendly closed-loop DBS monitoring}

Another demand is providing a more patient-friendly closed-loop DBS device by making commands through wireless data communications for home care facilities. Early detection of DBS side effects could result in immediate action of neurologists in modifying stimulation parameters. This cannot be achieved unless through a long-term and ongoing monitoring of the disease state. Even in the closedloop DBS where programming is done automatically, the need for monitoring the stimulator related side-effects exists [116]. Apart from the stimulator's side effects, the battery capacity needs to be monitored after implantation to avoid hazards of battery depletion [116].

Upon development of information technology, telemedicine is progressively applied for various medical applications. However, only a few programs have focused on connecting DBS devices to the hospital networks. A web-based platform (WebBioBank) for neurophysiological DBS data collection has been developed for integration of PD clinical data [117, 118]. This system creates a reference guideline for home care and monitoring of DBS patients. It is capable of connecting to patient's smartphone and safely share the information to improve the care quality for DBS patients. However, such an integrated system is in the early stages of development and requires further research to become available not only to PD patients but also to other treatable disorders via DBS. In another study, Chen et al. [119] first identified the DBS telemedicine requirements through a questionnaire responded by 22 patients and 9 neurosurgeons. The results indicated that providing of an urgent remote adjustment was needed. Moreover, the preferred communication method was the video telehealth interaction. According to their findings, adding an interaction mode to the DBS systems was proposed. Then, they successfully tested the functionality of the idea on three patients.

\section{Commercial closed-loop DBS devices}

DBS devices have been in commercial use since 1997 when the food and drug administration (FDA) organization approved the utilization of neurostimulator devices for tremor treatment [120]. Since then, they have been also approved for other disorders such as PD in 2002 [121], dystonia in 2003 [122] and obsessive-compulsive disorder (OCD) in 2009 [123]. The Activa system by Medtronic (Minneapolis, MN, USA) is a widespread open-loop DBS device. However, other companies such as Boston Scientific and St. Jude Medical also manufactured comparable products.

The study of the relevant literature shows that there is currently only one commercially available brain stimulation system that provides closed-loop DBS (The closed-loop RNS system by NeuroPace for closed-loop cortical stimulation). The closed-loop RNS system can stimulate depth and/or cortical tissues of the brain using two different types of leads. It is a responsive battery powered closed-loop DBS capable of continuous sensing of ECoG potentials. The RNS system has received FDA approval to be used as an alternative option for treatment of drug-resistant epilepsy patients [124]. Upon recognition of a predefined pattern relating to seizure in the recorded signal, the stimulator is activated to remove the pattern. This device stores segments of ECoG potentials. In addition, it can be programmed to store information about the detected electrographic events (e.g. the date and time of seizure occurrence).

Another commercial closed-loop DBS system is the Activa $\mathrm{PC}+\mathrm{S}$ which is an implantable sensing-stimulating DBS device manufactured by Medtronic. This device has been designed for investigational purposes. The $\mathrm{PC}+\mathrm{S}$ system can provide concurrent LFP sensing and stimulation. However, it does not use the sensed information for adjusting the stimulation pulses [125], unless the user compiles a program into the device and closes the feedback loop. If Medtronic provides an upgrade for the $\mathrm{PC}+\mathrm{S}$ system with all the necessary programs for closing the feedback loop, then, the Activa PC $+\mathrm{S}$ system can function as a complete closed-loop DBS device.

\section{Discussions}

\section{Weakness of open-loop DBS devices}

Once a conventional DBS system is implanted, the stimulation parameters need to be defined. The procedure in which the optimum stimulation parameters are defined per each subject is a trial-and-error-based programming task that is carried out by clinicians. Using a set of guidelines, the stimulation parameters are changed based on the observable behavioral responses to the previous stimulation parameters [126]. Unfortunately, this procedure is a subject-dependent and timeconsuming task that may frustrates both the clinician and patient.

Although the neurologists reprogram the open-loop DBS to enhance the therapeutic results, this reprogramming is not an optimal method. Even by achieving an optimum set of parameters in one effort, there is no guarantee that the symptom relief response will last long due to the brain neural plasticity. Neural plasticity usually occurs after prolonged DBS resulting in reduced efficacy over time. For instance, Ricchi et al. [127] observed that the gait improvement obtained by switching the stimulation frequency from $130 \mathrm{~Hz}$ to $80 \mathrm{~Hz}$ was not achievable at follow-up sessions. Occasionally, it is feasible to recapture the efficacy of openloop DBS using a coordinated reset (CR) stimulation [128, 129], which is a short-duration stimulation interval for rewiring of neuronal networks [130]. Many computational studies reported optimization of CR stimulation so that long lasting therapeutic effects can be achieved $[64,65,131]$. The 
results were obtained in rat hippocampal slices [132], MPTP-treated monkeys [133, 134], human PD patients [135] as well as in tinnitus patients [136, 137]. The outcomes confirm the robustness of CR open-loop stimulation for the treatment of neurological disorders characterized by abnormal neuronal synchronization [131]. Note, the application of CR stimulation is not limited to open-loop DBS, and can also be used with closed-loop DBS in a demandcontrolled manner $[128,129,138]$ to fight against induced neural plasticity over time.

Furthermore, the open-loop programming procedure may not be practicable for all kind of neural disorders. For instance, the therapeutic response to the programming of stimulation parameters could take several weeks to finalize for depression or dystonia [71, 139], while being instantly evident for epilepsy, tremor, and PD [71]. Taking a longer time for appearance of the therapeutic response makes it hard for neurologists to decide whether the adjusted parameters are superior to the previous settings or not. Therefore, the open-loop programming could be inefficient for certain neurological disorders.

Another weakness of open-loop DBS devices is that its frequent programming sessions incur additional costs to the patients. Patients normally don't like the numerous clinic visits required for adjustments of parameters to optimize their symptom relief response. In addition, reprogramming of open-loop DBS devices needs regular involvement of clinical experts. Therefore, this could be an extra burden on clinics to employ and instruct clinical staffs to train them skills in open-loop DBS programming [140].

In addition, open-loop DBS devices are faced with the issues associated with battery life due to their continuous and non-stop stimulation pulses. One issue is that battery replacement requires a surgery which imposes potential risks (e.g. surgery risks, anesthesia risks) to the patient [3].

In summary, open-loop DBS therapy has several weaknesses including: (1) subject dependency, (2) timeconsuming programming, (3) lack of dynamical adjustment of stimulation parameters, (4) frequent visits to clinic for programming, (5) extra costs in visits to clinic, (6) inefficiency for some neurological disorders (e.g. disorders with a lag response to the stimulation), (7) regular involvement of programming experts, and (8) short battery life in battery-operated devices. Hence, the open-loop programming of DBS devices is not optimal method and might not result in the best therapeutic effects.

\section{Comparison of closed-loop DBS devices}

A comparison of the existing closed-loop DBS devices is presented in Table 2. It provides an evaluation of the existing device from different technical standpoints. Although these devices have common functional features, they incorporate different monitoring and stimulation designs, control methods, and internal features. Some of these devices close the feedback loop by turning the stimulation on and off known as ondemand stimulation [31, 32, 104, 141]. The stimulation is delivered only when the abnormal phase of biomarker is detected. While other devices take a step forward and update at least one stimulation parameter based on the biomarker state, usually pulse amplitude [86, 102, 114]. The existing closed-loop DBS devices (as compared in Table 2) employ only one biomarker in the control unit to decide on the adjustment of stimulation parameters. While some of the devices can be used to record more than one biomarker (e.g. LFPs and APs), but they cannot detect and process multiple biomarkers concurrently. Therefore, only one biomarker is used as a feedback to control the DBS pulse generator. Using only one biomarker to close the feedback loop has the disadvantage of being affected by noise and artifacts. Occasionally, due to the device internal or external environmental conditions, the biomarker and the electrodes can be affected by some unexpected noises and artifacts. These unexpected conditions include, but not limited to, magnetic field interactions, induced electric currents, temperature variations, device functional disruptions $[142,143]$. If the device is affected by one of the stated conditions, the biomarker will be affected and a wrong decision will be made by the controller. Consequently, the DBS unit will adjust the stimulation parameters unrelated to the variations in the patient clinical state. Being unrelated to the disease may lead to either opposing pathological effects or insufficient treatment. Moreover, the neurons may get damaged because of the incorrect adjustment of the stimulation parameters [144]. One solution is to provide the controller with multiple feedback signals [145]. Whenever a fault occurs in one biomarker sensing path, there will be another path to steer the closed-loop operation. Therefore, this could increase the robustness of the closed-loop DBS device and decrease the chance of inaccurate adjustment of stimulation parameters.

\section{Issues with closed-loop DBS devices}

While closed-loop DBS has shown superiority in improvement of clinical outcomes [2], it faces several issues (summarized in Fig. 5). One of the challenges is finding a reliable biomarker linked to the patient's symptoms. Although several physiological and biochemical biomarkers have been introduced for closing the feedback loop, some of the biomarkers such as LFPs, AP, ECoG, and EEG are affected by the stimulation pulses. Thus, the detection of the biomarker is affected requiring sophisticated artifact removing circuitry to improve the detection of the biomarker. Thus far, several artifact rejection 


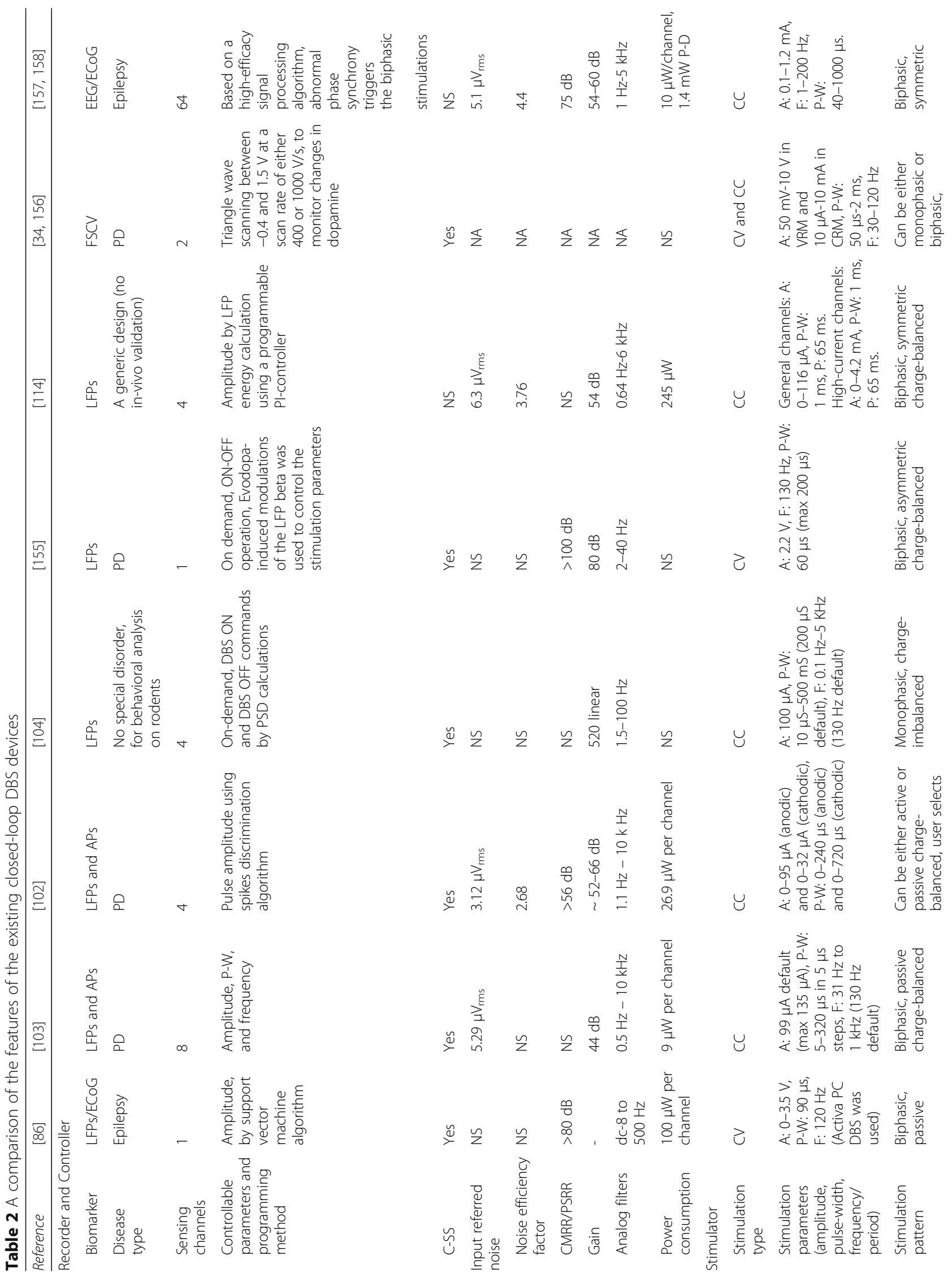




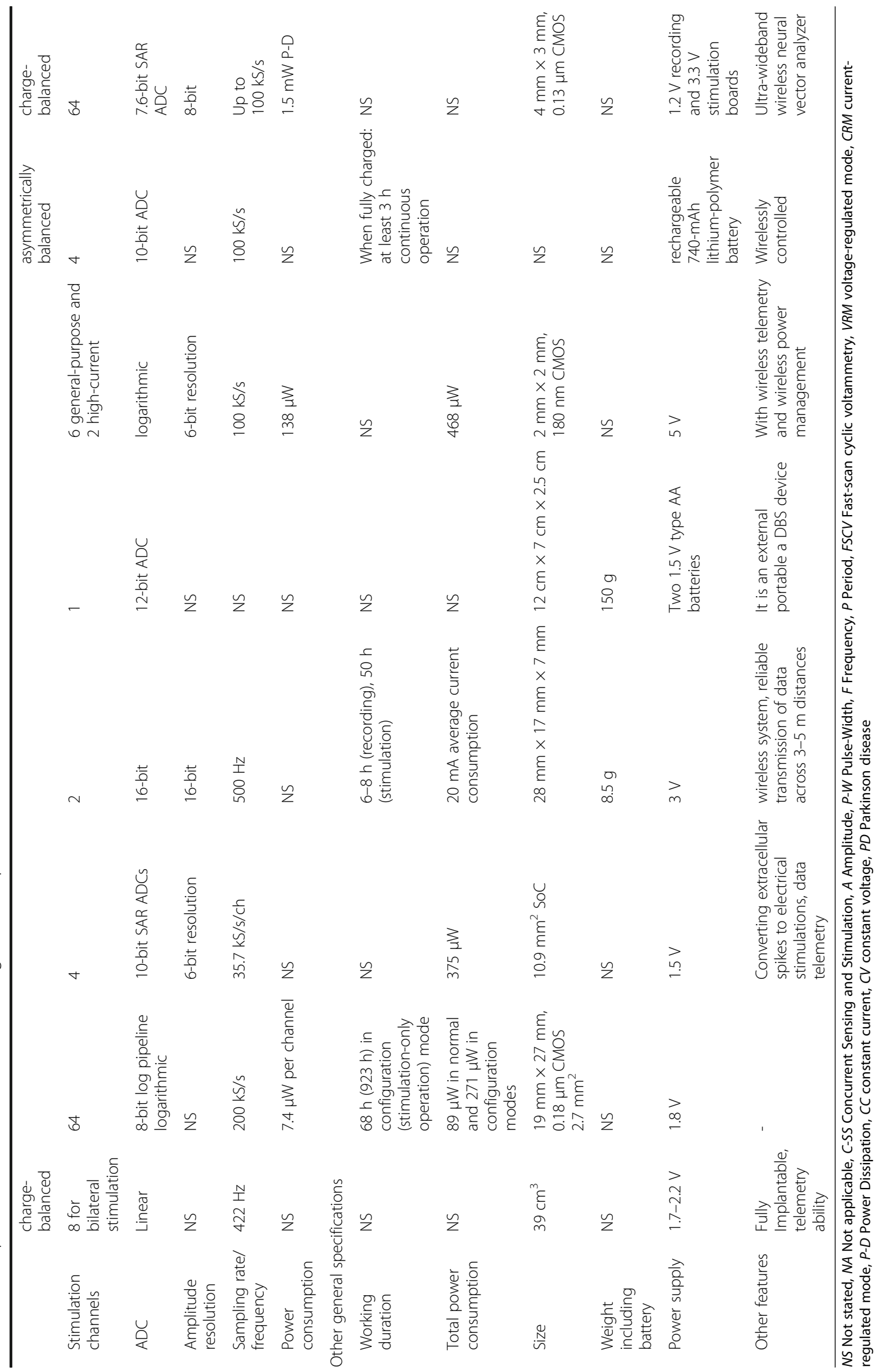




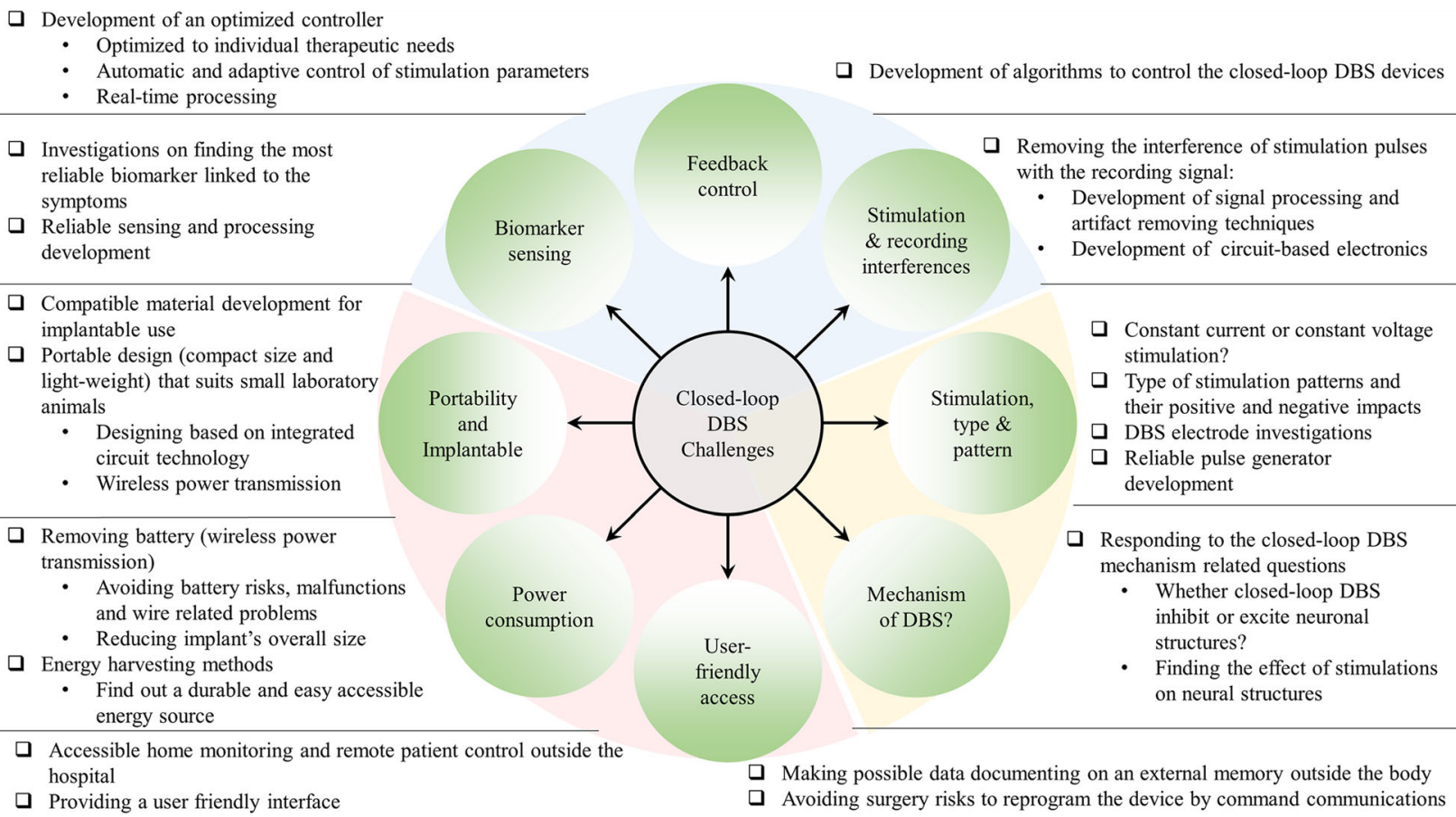

Investigations on finding the most reliable biomarker linked to the symptoms

Reliable sensing and processing development

C Compatible material development for implantable use

- Portable design (compact size and light-weight) that suits small laboratory animals

- Designing based on integrated circuit technology

- Wireless power transmission

Removing battery (wireless power transmission)

- Avoiding battery risks, malfunctions and wire related problems

- Reducing implant's overall size

Energy harvesting methods

- Find out a durable and easy accessible energy source

Accessible home monitoring and remote patient control outside the hospital

- Providing a user friendly interface

D Development of algorithms to control the closed-loop DBS devices

Removing the interference of stimulation pulses with the recording signal:

- Development of signal processing and artifact removing techniques

- Development of circuit-based electronics

Fig. 5 Closed-loop DBS research challenges. These challenges are classifiable in three major parts including monitoring issues (blue part), stimulation challenges (yellow part), and design expectation concerns (red part)

techniques have been developed to tackle the impact of stimulation on biomarker recording [84, 86, 87, 146]; however, the issue has been resolved partially in the current closed-loop DBS devices.

Another challenge is related to the type of stimulation. The clinical difference between $\mathrm{CC}$ and $\mathrm{CV}$ stimulation needs to be investigated to find out which approach is more advantageous. The pattern of stimulation is another concern due to the fact that a charge-imbalanced pulse would cause damages to the brain tissue. Most of the available market-based open-loop and closed-loop DBS systems use a passive charge-balancing scheme, requiring further investigation and upgrade to an active chargebalancing scheme in order to avoid the weaknesses of the passive charge-balancing as described by Sooksood et al. [89]. On one hand, because of safety issues, the pattern of stimulation pulses needs to be biphasic. On the other hand, biphasic pulses can alter neural activities and lead to suppressing effects on the action potentials. As a consequence, more studies need to explore the clinical impact of charge-balanced and charge-imbalanced stimulation pulses in the closed-loop DBS devices. In addition, the role of stimulation patterns will need to be investigated to be able to answer the remaining questions on the mechanisms and benefits of DBS.

In order to evaluate the effectiveness of the closedloop DBS devices or examine new applications, the devices need to be validated in-vivo. Due to the small size of most of laboratory animals and also because of the need for long-term experiments, the devices need to have compact size and light-weight, be portable and implantable, and have a tetherless (wireless) configuration to not disturb animal freedom of movement. These are considered as other challenges in designing a closedloop DBS device. Since closed-loop DBS devices have recording circuitry, the total size and weight of the device may be affected.

In addition, in terms of power consumption, closedloop DBS devices are expected to consume less power compared with their open-loop counterparts. The reason is that closed-loop devices deliver electrical stimulation based on the state of the brain. Therefore, these devices remain less active over a course of time which results in a reduction of the electrical energy delivered to the brain [4]. However, closed-loop devices have extra recording and processing circuits that could increase the overall power consumption of the device. Therefore, the potential power saving of these devices would depend on the use of low-power recording and analysis circuits.

Although closed-loop DBS devices have a control algorithm that automatically modifies the stimulation parameters according to the patient's brain state, they may still require regular assessments of the stimulator related sideeffects and, if necessary, reprogramming of the control algorithm for minimization of adverse-effects. Developing an optimal control algorithm and/or discovering an 
optimum biomarker individualized to each patient can take a long time. Therefore, the patients are required to pay extra costs associated with the service. Designing a patient-friendly closed-loop DBS device is another requirement that deserves consideration. This means that providing wireless data and command communications for home care applications is needed to reduce costs and improve care quality.

There are also other concerns associated with the closed-loop DBS therapy, one of them being the effect of pharmacological approaches on the nature and magnitude of measured biomarkers of interest. Simultaneous medication (either oral or injection) with DBS therapy could affect the neuronal activities and change the amplitude of the recorded biomarker. As an example, it has been observed that beta activity is suppressed with levodopa treatment in PD patients [80]. In addition, it has been also demonstrated that DBS could suppress beta activity in off-medication state [147]. However, the patterns of beta oscillations following DBS, differ from those after medication [148]. Simultaneous pharmacological intervention could potentially attempt to change the closed-loop DBS results when applied. Besides the pharmacological intervention, the interaction of progressive severity of the disease may affect the measured biomarkers. Very little work has been done to demonstrate a correlation between a particular biomarker and disease severity over time. In the presence of an opposite correlation, this factor might pose a problem for the use of biomarker in the feedback loop. Therefore, it reinforces the use of individualized biomarkers based on patient's clinical phenotype or use of combination of biomarkers [48].

Another concern is alleviation of a range of disease symptoms using a single biomarker (or multiple biomarkers). Take PD for instance, each patient may experience different primary (e.g. tremor, bradykinesia, rigidity, postural instability, etc.) or secondary (e.g. freezing, micrographia, mask-like expression, unwanted accelerations) motor symptoms [149]. Some of these symptoms are promoted by different pathophysiological neuronal networks and, therefore, may require different biomarkers to detect. Thus, amelioration of a range of PD motor symptoms through least a number of biomarkers need further and future investigations. Viability of biomarker recordings over the course of several years is another concern. The choice of sensing methodology, electrode materials and components in the implants, could potentially increase the viability of biomarker recordings. The use of LFPs, which could be recorded from the same lead used to induce DBS, also offers a more stable biomarker measurements. However, selection of a biomarker that requires a separate recording path from those implanted for DBS may not assure the viability of recordings and require further investigations.

\section{Towards commercializing closed-loop DBS devices}

The promising clinical effects of open-loop DBS have been demonstrated, indicating DBS as a pioneer technology and treatment option to serve neurological patients. Moreover, the significant increase in the number of commercial open-loop DBS devices represents the approach effectiveness. However, like other commercial devices, DBS needs to be automated and modernized. Currently, in the open-loop DBS approach, the stimulation parameters are periodically adjusted by neurologists and the parameters remain constant in the intervals of two subsequent visits regardless of any variations in the patient's clinical state. Therefore, it may cause brain overstimulation or produce fewer benefits because of insufficient stimulations. Closed-loop DBS can eliminate brain overstimulation and provide maximum stimulation efficiency for those in need of DBS therapy.

Despite the advancements in the closed-loop DBS devices, most current commercial DBS systems operate in an open-loop manner. To date, only one commercial closed-loop DBS device has been manufactured (the RNS system). However, life expectancy of IPGs is not very good for the RNS system. The battery charge is rapidly depleted when stimulation is used heavily, and the control algorithm is currently conventional based on on-demand (On-Off) approach [125]. A closed-loop DBS device requires an adaptive algorithm to learn and optimize the stimulation parameters according to the brain clinical state. Owing to the fact that each patient is different, such an algorithm may need to be patient dependent.

\section{Conclusion}

Recent advances in the closed-loop DBS systems provide a brighter future for patients in need of DBS treatment. The next generation of DBS devices is expected to be fully and automatically programmable, compatible with biomarker variations, and flexible in stimulation type and pattern, to yield greater benefits and fewer side effects. Since a new technology has to be accessible to all patients over the world, the next closed-loop designs need to be inexpensive to become available even in developing countries. In addition, it can be expected that the device operates based on multiple biomarkers to decrease the chance of malfunction and guarantee the robustness of the closed-loop DBS operation. Engineers need to provide a balance and tradeoff among the device features by considering the clinical effectiveness, and the technological costs of the device. However, whether the future advancements in the closedloop DBS devices can result in a better life and efficient care for their users need to be yet demonstrated. Alongside all the advancements and benefits that closed-loop DBS devices can bring to the patients, other brain stimulation methods e.g. optogenetics and ultrasonic stimulations are also receiving attention by research communities. 


\section{Abbreviations}

AC: Alternating current; ADC: Analog to digital converter; AP: Action potential; BCI: Brain computer interface; CC: Constant-current-controlled; CMOS: Complementary metal-oxide-semiconductor; CMRR: Common mode rejection ratio; CR: Coordinated-reset; CV: Constant-voltage-controlled; DBS: Deep brain stimulation; DC: Direct current; ECoG: Electrocorticogram; EEG: Electroencephalogram; EMG: Electromyogram; FDA: Food and drug administration; FSCV: Fast-scan cyclic voltammetry; IC: Integrated circuit; LFP: Local field potential; MPTP: 1-methyl-4-phenyl-1,2,3,6-tetrahydropyridine; OCD: Obsessive-compulsive disorder; PD: Parkinson's disease; PI: Proportionalintegral; PID: Proportional-integral-derivative; RNS: Responsive neurostimulator; SCS: Spinal cord stimulation; SoC: System-on-chip; STN: Subthalamic nucleus; VNS: Vagus nerve stimulation

\section{Acknowledgements}

Not Applicable.

\section{Funding}

Not Applicable.

\section{Availability of data and materials}

Data sharing not applicable to this review article as no datasets were generated or analysed during the current study

\section{Authors' contributions}

$M P$ and $A B$ carried out the review of the other works in the area. MP and $A B$ participated in the sequence alignment. MP wrote the first draft of the manuscript. AB reviewed the first draft. All authors read and approved the final manuscript.

\section{Ethics approval and consent to participate}

Not Applicable.

\section{Competing interests}

The authors declare that they have no competing interests.

\section{Publisher's Note}

Springer Nature remains neutral with regard to jurisdictional claims in published maps and institutional affiliations.

Received: 9 June 2017 Accepted: 4 August 2017

\section{Published online: 11 August 2017}

\section{References}

1. Hamani C, Richter E, Schwalb JM, Lozano AM. Bilateral subthalamic nucleus stimulation for Parkinson's disease: a systematic review of the clinical literature. Neurosurgery. 2005;56:1313-1321; discussion 1321-4.

2. Rosin B, Slovik M, Mitelman R, Rivlin-Etzion M, Haber SN, Israel Z, et al. Closed-loop deep brain stimulation is superior in ameliorating parkinsonism. Neuron. 2011;72:370-84

3. Little S, Pogosyan A, Neal S, Zavala B, Zrinzo L, Hariz M, et al. Adaptive deep brain stimulation in advanced Parkinson disease. Ann Neurol. 2013;74:449-57.

4. Wu H, Ghekiere H, Beeckmans D, Tambuyzer T, van Kuyck K, Aerts J-M, et al. Conceptualization and validation of an open-source closed-loop deep brain stimulation system in rat. Sci Rep. 2015;4:9921.

5. Hess CW, Vaillancourt DE, Okun MS. The temporal pattern of stimulation may be important to the mechanism of deep brain stimulation. Exp Neurol. 2013;247:296-302

6. S. Chiken and A. Nambu, "Mechanism of Deep Brain Stimulation: Inhibition, Excitation, or Disruption?" Neuroscientist. 2016;22:313-22.

7. Herrington TM, Cheng JJ, Eskandar EN. Mechanisms of deep brain stimulation. J Neurophysiol. 2016;115:19-38.

8. Broccard FD, Mullen T, Chi YM, Peterson D, Iversen JR, Arnold M, et al. Closed-loop brain-machine-body interfaces for noninvasive rehabilitation of movement disorders. Ann Biomed Eng. 2014:42:1573-93.

9. Hebb AO, Zhang JJ, Mahoor MH, Tsiokos C, Matlack C, Chizeck HJ, et al. Creating the feedback loop: closed-loop Neurostimulation. Neurosurg Clin N Am. 2014;25:187-204.

10. Hariz M. Deep brain stimulation: new techniques. Parkinsonism Relat Disord 2014;20(Supplement 1):S192-6.
11. Hosain MK, Kouzani A, Tye S. Closed loop deep brain stimulation: an evolving technology. Australas Phys Eng Sci Med. 2014;37:619-34.

12. Priori A, Foffani G, Rossi L, Marceglia S. Adaptive deep brain stimulation (aDBS) controlled by local field potential oscillations. Exp Neurol. 2013;245:77-86.

13. Shah RS, Chang SY, Min HK, Cho ZH, Blaha CD, Lee KH. Deep brain stimulation: technology at the cutting edge. J Clin Neurol. Dec 2010;6:167-82.

14. Priori A. Technology for deep brain stimulation at a gallop. Mov Disord. 2015;30:1206-12.

15. Mclntyre CC, Chaturvedi A, Shamir RR, Lempka SF. Engineering the next generation of clinical deep brain stimulation technology. Brain Stimul. 2015; 8:21-6.

16. Arlotti M, Rosa M, Marceglia S, Barbieri S, Priori A. The adaptive deep brain stimulation challenge. Parkinsonism Relat Disord. 2016;28:12-7.

17. Osorio I, Frei MG, Manly BF, Sunderam S, Bhavaraju NC, Wilkinson SB. An introduction to contingent (closed-loop) brain electrical stimulation for seizure blockage, to ultra-short-term clinical trials, and to multidimensional statistical analysis of therapeutic efficacy. J Clin Neurophysiol. 2001;18:533-44.

18. A. Murro, Y. Park, D. Greene, J. Smith, P. Ray, D. King, et al., "Closed-loop neurostimulation in patient with intractable epilepsy," J Clinical Neurophysiology. 2003;20:222.

19. A. Murro, Y. Park, G. Bergey, E. Kossoff, E. Ritzl, S. Karceski, et al., "Multicenter study of acute responsive stimulation in patients with intractable epilepsy," Epilepsia. 2003;44(Supplement 9):326.

20. Sun FT, Morrell MJ, Wharen RE Jr. Responsive cortical stimulation for the treatment of epilepsy. Neurotherapeutics. 2008;5:68-74.

21. Schulze-Bonhage A. Deep brain stimulation: a new approach to the treatment of epilepsy. Dtsch Arztebl Int. 2009;106:407-12.

22. Lettieri C, Rinaldo S, Devigili G, Pauletto G, Verriello L, Budai R, et al. Deep brain stimulation: subthalamic nucleus electrophysiological activity in awake and anesthetized patients. Clin Neurophysiol. 2012;123:2406-13.

23. P. Afshar, D. Moran, A. Rouse, W. Xuan, and T. Denison, "Validation of chronic implantable neural sensing technology using electrocorticographic (ECoG) based brain machine interfaces," 2011 5th International IEEE/EMBS Conference on Neural Engineering, Cancun, 2011, pp. 704-707.

24. S. E. Qasim, C. de Hemptinne, N. C. Swann, S. Miocinovic, J. L. Ostrem, and P. A. Starr, "Electrocorticography reveals beta desynchronization in the basal ganglia-cortical loop during rest tremor in Parkinson's disease," Neurobiol Dis, vol. 86, pp. 177-186, 2//2016.

25. R. Hyo-Gyuem, J. Jaehun, J. A. Fredenburg, S. Dodani, P. Patil, and M. P. Flynn, "A wirelessly powered log-based closed-loop deep brain stimulation SoC with two-way wireless telemetry for treatment of neurological disorders," 2012 Symposium on VLSI Circuits (VLSIC), Honolulu, HI, 2012, pp. 70-71.

26. Abosch A, Lanctin D, Onaran I, Eberly L, Spaniol M, Ince NF. Long-term recordings of local field potentials from implanted deep brain stimulation electrodes. Neurosurgery. 2012;71:804-14.

27. Giannicola G, Rosa M, Servello D, Menghetti C, Carrabba G, Pacchetti C, et al. Subthalamic local field potentials after seven-year deep brain stimulation in Parkinson's disease. Exp Neurol. 2012;237:312-7.

28. Neumann WJ, Huebl J, Brucke C, Gabriels L, Bajbouj M, Merkl A, et al. Different patterns of local field potentials from limbic DBS targets in patients with major depressive and obsessive compulsive disorder. Mol Psychiatry. 2014;19:1186-92.

29. Swann N, Poizner H, Houser M, Gould S, Greenhouse I, Cai W, et al. Deep brain stimulation of the subthalamic nucleus alters the cortical profile of response inhibition in the beta frequency band: a scalp EEG study in Parkinson's disease. J Neurosci. 2011;31:5721-9.

30. Graupe D, Basu I, Tuninetti D, Vannemreddy P, Slavin KV. Adaptively controlling deep brain stimulation in essential tremor patient via surface electromyography. Neurol Res. 2010;32:899-904.

31. Basu I, Graupe D, Tuninetti D, Shukla P, Slavin KV, Metman LV, et al. Pathological tremor prediction using surface electromyogram and acceleration: potential use in 'ON-OFF' demand driven deep brain stimulator design. J Neural Eng. 2013;10:036019.

32. Yamamoto $T$, Katayama $Y$, Ushiba J, Yoshino $H$, Obuchi $T$, Kobayashi $K$, et al. On-demand control system for deep brain stimulation for treatment of intention tremor. Neuromodulation. 2013:16:230-235; discussion 235.

33. Hilliard JD, Frysinger RC, Elias WJ. Effective subthalamic nucleus deep brain stimulation sites may differ for tremor, bradykinesia and gait disturbances in Parkinson's disease. Stereotact Funct Neurosurg. 2011;89:357-64. 
34. Chang SY, Kimble CJ, Kim I, Paek SB, Kressin KR, Boesche JB, et al. Development of the Mayo investigational Neuromodulation control system: toward a closed-loop electrochemical feedback system for deep brain stimulation. J Neurosurg. 2013;119:1556-65.

35. Sarnthein J, Jeanmonod D. High thalamocortical theta coherence in patients with Parkinson's disease. J Neurosci. 2007;27:124-31.

36. Schalk G, Leuthardt EC. Brain-computer interfaces using electrocorticographic signals. IEEE Rev Biomed Eng. 2011:4:140-54.

37. Burle B, Spieser L, Roger C, Casini L, Hasbroucq T, Vidal F. Spatial and temporal resolutions of EEG: is it really black and white? A scalp current density view. Int J Psychophysiol. 2015;97:210-20.

38. Schwartz AB, Cui XT, Weber DJ, Moran DW. Brain-controlled interfaces: movement restoration with neural prosthetics. Neuron. 2006;52:205-20.

39. Ball T, Kern M, Mutschler I, Aertsen A, Schulze-Bonhage A. Signal quality of simultaneously recorded invasive and non-invasive EEG. Neurolmage. 2009; 46:708-16.

40. Leuthardt EC, Freudenberg Z, Bundy D, Roland J. Microscale recording from human motor cortex: implications for minimally invasive electrocorticographic brain-computer interfaces. Neurosurg Focus. 2009;27:E10.

41. Slutzky MW, Jordan LR, Krieg T, Chen M, Mogul DJ, Miller LE. Optimal spacing of surface electrode arrays for brain-machine interface applications. J Neural Eng. 2010;7:26004.

42. Harrison RR, Watkins PT, Kier RJ, Lovejoy RO, Black DJ, Greger B, et al. A lowpower integrated circuit for a wireless 100-electrode neural recording system. Solid-State Circuits IEEE J. 2007;42:123-33.

43. Holleman J, Zhang F, Otis B. Ultra Low-power Integrated Circuit Design for Wireless Neural Interfaces. New York Springer Science+Business Media, LLC, 2011.

44. János AP, Mark LH, Wasim QM, Sydney C, Emad E, Gerhard F, et al. Intra-day signal instabilities affect decoding performance in an intracortical neural interface system. J Neural Eng. 2013;10:036004.

45. Paek S, Knight EJ, Chang S-Y, Lujan JL, Jang DP, Bennet KE, et al. Dopamine measurement during prolonged deep brain stimulation: a proof-of-principle study of paired pulse voltammetry. Biomed Eng Lett. 2013;3:22-31.

46. Buzsaki G, Anastassiou CA, Koch C. The origin of extracellular fields and currents-EEG, ECoG, LFP and spikes. Nat Rev Neurosci. 2012;13:407-20.

47. Kajikawa Y, Schroeder CE. How local is the local field potential? Neuron. 2011;72:847-58.

48. Little S, Brown P. What brain signals are suitable for feedback control of deep brain stimulation in Parkinson's disease? Ann N Y Acad Sci. 2012;1265:9-24

49. Einevoll GT, Kayser C, Logothetis NK, Panzeri S. Modelling and analysis of local field potentials for studying the function of cortical circuits. Nat Rev Neurosci. 2013;14:770-85.

50. W. Deeb, J. J. Giordano, P. J. Rossi, A. Y. Mogilner, A. Gunduz, J. W. Judy, et al., "Proceedings of the Fourth Annual Deep Brain Stimulation Think Tank: A Review of Emerging Issues and Technologies," Frontiers in Integrative Neuroscience, 2016;10:38.

51. J. Volkmann, "Deep brain stimulation for the treatment of Parkinson's disease," J Clin Neurophysiol, vol. 21, pp. 6-17, Jan-Feb 2004

52. Hunka K, Suchowersky O, Wood S, Derwent L, Kiss ZH. Nursing time to program and assess deep brain stimulators in movement disorder patients. J Neurosci Nurs. 2005;37:204-10.

53. Kupsch A, Tagliati M, Vidailhet M, Aziz T, Krack P, Moro E, et al. Early postoperative management of DBS in dystonia: programming, response to stimulation, adverse events, medication changes, evaluations, and troubleshooting. Mov Disord. 2011;26(Suppl 1):S37-53.

54. K. Ganguly and M. M. Poo, "Activity-dependent neural plasticity from bench to bedside," Neuron, vol. 80, pp. 729-741, Oct 302013.

55. M. G. Rosenblum and A. S. Pikovsky, "Controlling synchronization in an ensemble of globally coupled oscillators," Phys Rev Lett, vol. 92, p. 114102 Mar 192004.

56. Rosenblum M, Pikovsky A. Delayed feedback control of collective synchrony: an approach to suppression of pathological brain rhythms. Phys Rev E Stat Nonlinear Soft Matter Phys. Oct 2004;70:041904.

57. C. Hauptmann, O. Popovych, and P. A. Tass, "Delayed feedback control of synchronization in locally coupled neuronal networks,"Neurocomputing, vol. 65, pp. 759-767, 2005/06/01/2005

58. C. Hauptmann, O. Popovych, and P. A. Tass, "Effectively desynchronizing deep brain stimulation based on a coordinated delayed feedback stimulation via several sites: a computational study," Biol Cybern, vol. 93, pp. 463-470, Dec 2005.

59. O. V. Popovych, C. Hauptmann, and P. A. Tass, "Effective desynchronization by nonlinear delayed feedback," Phys Rev Lett, vol. 94, p. 164102, Apr 292005.

60. O. V. Popovych, C. Hauptmann, and P. A. Tass, "Control of neuronal synchrony by nonlinear delayed feedback," Biol Cybern, vol. 95, pp. 69-85, 2006/07/01 2006

61. I. Z. Kiss, C. G. Rusin, H. Kori, and J. L. Hudson, "Engineering complex dynamical structures: sequential patterns and desynchronization," Science, vol. 316, pp. 1886-1889, Jun 292007.

62. O. V. Popovych and P. A. Tass, "Synchronization control of interacting oscillatory ensembles by mixed nonlinear delayed feedback," Phys Rev E, vol. 82, p. 026204, 08/09/2010.

63. Tukhlina N, Rosenblum M, Pikovsky A, Kurths J. Feedback suppression of neural synchrony by vanishing stimulation. Phys Rev E Stat Nonlinear Soft Matter Phys. Jan 2007:75:011918.

64. Popovych OV, Lysyansky B, Rosenblum M, Pikovsky A, Tass PA. Pulsatile desynchronizing delayed feedback for closed-loop deep brain stimulation. PLoS One. 2017;12:e0173363.

65. O. V. Popovych, B. Lysyansky, and P. A. Tass, "Closed-loop deep brain stimulation by pulsatile delayed feedback with increased gap between pulse phases," Sci Rep, vol. 7, p. 1033, 2017/04/21 2017.

66. Santaniello S, Fiengo G, Glielmo L, Grill WM. Closed-loop control of deep brain stimulation: a simulation study. IEEE Trans Neural Syst Rehabil Eng. 2011;19:15-24.

67. Pyragas K, Popovych OV, Tass PA. Controlling synchrony in oscillatory networks with a separate stimulation-registration setup. EPL (Europhysics Letters). 2007;80:40002

68. Gorzelic P, Schiff SJ, Sinha A. Model-based rational feedback controller design for closed-loop deep brain stimulation of Parkinson's disease. J Neural Eng. 2013;10:026016.

69. E. M. Dunn and M. M. Lowery, "Simulation of PID control schemes for closedloop deep brain stimulation," 2013 6th International IEEE/EMBS Conference on Neural Engineering (NER), San Diego, CA, 2013, pp. 1182-1185.

70. Su F, Wang J, Deng B, Wei XL, Chen YY, Liu C, et al. Adaptive control of Parkinson's state based on a nonlinear computational model with unknown parameters. Int J Neural Syst. 2015;25:1450030

71. P. J. Grahn, G. W. Mallory, O. U. Khurram, B. M. Berry, J. T. Hachmann, A. J. Bieber, et al., "A neurochemical closed-loop controller for deep brain stimulation: toward individualized smart neuromodulation therapies," Frontiers in Neuroscience, 2014:8:169.

72. Meidahl AC, Tinkhauser G, Herz DM, Cagnan H, Debarros J, Brown P. Adaptive deep brain stimulation for movement disorders: the long road to clinical therapy. Mov Disord. 2017;32:810-9.

73. Michmizos KP, Frangou P, Stathis P, Sakas D, Nikita KS. Beta-band frequency peaks inside the subthalamic nucleus as a biomarker for motor improvement after deep brain stimulation in Parkinson's disease. IEEE J Biomed Health Inform. 2015:19:174-80.

74. Davidson CM, de Paor AM, Cagnan H, Lowery MM. Analysis of oscillatory neural activity in series network models of Parkinson's disease during deep brain stimulation. IEEE Trans Biomed Eng. 2016;63:86-96.

75. E. J. Quinn, Z. Blumenfeld, A. Velisar, M. M. Koop, L. A. Shreve, M. H. Trager et al., "Beta oscillations in freely moving Parkinson's subjects are attenuated during deep brain stimulation," Mov Disord, vol. 30, pp. 1750-1758, Nov 2015.

76. S. Little, A. Pogosyan, S. Neal, L. Zrinzo, M. Hariz, T. Foltynie, et al, "Controlling Parkinson's Disease With Adaptive Deep Brain Stimulation," J Vis Exp. 2014;(89):51403.

77. S. Little, M. Beudel, L. Zrinzo, T. Foltynie, P. Limousin, M. Hariz, et al., "Bilateral adaptive deep brain stimulation is effective in Parkinson's disease. J Neurol Neurosurg PsychiatrySeptember 30, 20152015.

78. M. Rosa, M. Arlotti, G. Ardolino, F. Cogiamanian, S. Marceglia, A. Di Fonzo, et al., "Adaptive deep brain stimulation in a freely moving Parkinsonian patient," Mov Disord, vol. 30, pp. 1003-1005, Jun 2015.

79. L. A. Johnson, S. D. Nebeck, A. Muralidharan, M. D. Johnson, K. B. Baker, and J. L. Vitek, "Closed-loop deep brain stimulation effects on Parkinsonian motor symptoms in a non-human primate - is Beta enough?," Brain Stimul, vol. 9, pp. 892-896, Nov - Dec 2016.

80. Ray NJ, Jenkinson N, Wang S, Holland P, Brittain JS, Joint C, et al. Local field potential beta activity in the subthalamic nucleus of patients with 
Parkinson's disease is associated with improvements in bradykinesia after dopamine and deep brain stimulation. Exp Neurol. 2008;213:108-13.

81. Beudel M, Brown P. Adaptive deep brain stimulation in Parkinson's disease. Parkinsonism Relat Disord. 2016;22(Supplement 1):S123-6.

82. D. Piña-Fuentes, S. Little, M. Oterdoom, S. Neal, A. Pogosyan, M. A. J. Tijssen, et al., "Adaptive DBS in a Parkinson's patient with chronically implanted DBS: A proof of principle," Movement Disorders, vol. 00, 2017.

83. Cagnan H, Pedrosa D, Little S, Pogosyan A, Cheeran B, Aziz T, et al. Stimulating at the right time: phase-specific deep brain stimulation. Brain. 2017;140:132-45

84. Rossi L, Foffani G, Marceglia S, Bracchi F, Barbieri S, Priori A. An electronic device for artefact suppression in human local field potential recordings during deep brain stimulation. J Neural Eng. 2007;4:96-106.

85. T. Al-ani, F. Cazettes, S. Palfi, and J. P. Lefaucheur, "Automatic removal of high-amplitude stimulus artefact from neuronal signal recorded in the subthalamic nucleus," J Neurosci Methods, vol. 198, pp. 135-146, May 152011.

86. Stanslaski S, Afshar P, Peng C, Giftakis J, Stypulkowski P, Carlson D, et al. Design and validation of a fully implantable, chronic, closed-loop Neuromodulation device with concurrent sensing and stimulation. IEEE Trans Neural Syst Rehabil Eng. 2012;20:410-21.

87. Zbrzeski A, Lewis N, Rummens F, Jung R, N'Kaoua G, et al. Low-gain, lownoise integrated neuronal amplifier for implantable Artifact-reduction recording system. J Low Power Electron Appl. 2013;3:279.

88. Piallat $B$, Chabardès $S$, Devergnas $A$, Torres $N$, Allain $M$, Barrat $E$, et al. MONOPHASIC BUT NOT BIPHASIC PULSES INDUCE BRAIN TISSUE DAMAGE DURING MONOPOLAR HIGH-FREQUENCY DEEP BRAIN STIMULATION. Neurosurgery. 2009:64:156-63.

89. Sooksood K, Stieglitz T, Ortmanns M. An active approach for charge balancing in functional electrical stimulation. IEEE Trans Biomed Circuits Syst. 2010:4:162-70.

90. Ewing SG, Porr B, Riddell J, Winter C, Grace AA. SaBer DBS: a fully programmable, rechargeable, bilateral, charge-balanced preclinical microstimulator for long-term neural stimulation. J Neurosci Methods. 2013;213:228-35.

91. Hyung-Min L, Hangue P, Ghovanloo M. A power-efficient wireless system with adaptive supply control for deep brain stimulation. IEEE J Solid-State Circuits. 2013;48:2203-16.

92. Baker KB, Zhang J, Vitek JL. Pallidal stimulation: effect of pattern and rate on bradykinesia in the non-human primate model of Parkinson's disease. Exp Neurol. 2011;231:309-13.

93. Dorval AD, Kuncel AM, Birdno MJ, Turner DA, Grill WM. Deep brain stimulation alleviates parkinsonian bradykinesia by regularizing pallidal activity. J Neurophysiol. 2010;104:911-21.

94. Lettieri C, Rinaldo S, Devigili G, Pisa F, Mucchiut M, Belgrado E, et al. Clinical outcome of deep brain stimulation for dystonia: constant-current or constant-voltage stimulation? A non-randomized study. Eur J Neurol. 2015;22:919-26.

95. Rosa M, Marceglia S, Servello D, Foffani G, Rossi L, Sassi M, et al. Time dependent subthalamic local field potential changes after DBS surgery in Parkinson's disease. Exp Neurol. Apr 2010;222:184-90.

96. Lempka SF, Miocinovic S, Johnson MD, Vitek JL, McIntyre CC. In vivo impedance spectroscopy of deep brain stimulation electrodes. J Neural Eng. 2009;6:-046001.

97. Sillay KA, Rutecki P, Cicora K, Worrell G, Drazkowski J, Shih JJ, et al. Longterm measurement of impedance in chronically implanted depth and subdural electrodes during responsive neurostimulation in humans. Brain Stimul. Sep 2013;6:718-26.

98. T. Cheung, M. Nuno, M. Hoffman, M. Katz, C. Kilbane, R. Alterman, et al., "Longitudinal impedance variability in patients with chronically implanted DBS devices," Brain Stimul, vol. 6, pp. 746-751, Sep 2013.

99. Satzer $D$, Lanctin $D$, Eberly $L E$, Abosch $A$. Variation in deep brain stimulation electrode impedance over years following electrode implantation. Stereotact Funct Neurosurg. 2014;92:94-102.

100. Ramirez de Noriega F, Eitan R, Marmor O, Lavi A, Linetzky E, Bergman H, et al. Constant current versus constant voltage subthalamic nucleus deep brain stimulation in Parkinson's disease. Stereotact Funct Neurosurg. 2015;93:114-21.

101. Beaulieu-Boire I, Fasano A. Current or voltage? Another Shakespearean dilemma. Eur J Neurol. 2015;22:887-8.

102. Azin M, Guggenmos DJ, Barbay S, Nudo RJ, Mohseni P. A battery-powered activity-dependent Intracortical microstimulation IC for brain-machine-brain Interface. IEEE J Solid-State Circuits. 2011;46:731-45.
103. Jongwoo L, Hyo-Gyuem R, Kipke DR, Flynn MP. A 64 channel programmable closed-loop Neurostimulator with 8 channel neural amplifier and logarithmic ADC. IEEE J Solid-State Circuits. 2010;45:1935-45.

104. R. C. Pinnell, J. Dempster, and J. Pratt, "Miniature wireless recording and stimulation system for rodent behavioural testing," J Neural Eng, vol. 12, p. 066015, Oct 152015.

105. Avestruz AT, Santa W, Carlson D, Jensen R, Stanslaski S, Helfenstine A, et al. A 5 uW/channel spectral analysis IC for chronic bidirectional brain-machine interfaces. IEEE J Solid-State Circuits. 2008;43:3006-24.

106. Verma N, Shoeb A, Bohorquez J, Dawson J, Guttag J, Chandrakasan AP. A micro-power EEG acquisition SoC with integrated feature extraction processor for a chronic seizure detection system. IEEE J Solid-State Circuits. 2010;45:804-16.

107. Fan Z, Mishra A, Richardson AG, Otis B. A low-power ECoG/EEG processing IC with integrated multiband energy extractor. IEEE Trans Circuits and Syst I. 2011;58:2069-82.

108. J. Dams, U. Siebert, B. Bornschein, J. Volkmann, G. Deuschl, W. H. Oertel, et al., "Cost-effectiveness of deep brain stimulation in patients with Parkinson's disease," Mov Disord, vol. 28, pp. 763-771, Jun 2013.

109. Hannan MA, Mutashar S, Samad SA, Hussain A. Energy harvesting for the implantable biomedical devices: issues and challenges. Biomed Eng Online. 2014;13:79.

110. M. K. Hosain, A. Kouzani, and S. Tye, "Multi-layer implantable antenna for closed loop deep brain stimulation system," 2012 International Symposium on Communications and Information Technologies (ISCIT), Gold Coast, QLD, 2012, pp. 643-648.

111. Hosain MK, Kouzani AZ, Tye SJ, Samad MF, Kale RP, Bennet KE, et al. Radio frequency energy harvesting from a feeding source in a passive deep brain stimulation device for murine preclinical research. Med Eng Phys. 2015;37:1020-6.

112. M. K. Hosain, A. Z. Kouzani, S. J. Tye, O. A. Abulseoud, and M. Berk, "Design and analysis of an antenna for wireless energy harvesting in a headmountable DBS device," 2013 35th Annual International Conference of the IEEE Engineering in Medicine and Biology Society (EMBC), Osaka, 2013, pp. 3078-3081.

113. Hosain MK, Kouzani AZ, Tye S, Kaynak A, Berk M. RF rectifiers for EM power harvesting in a deep brain stimulating device. Australas Phys Eng Sci Med. 2015;38:157-72.

114. Hyo-Gyuem R, Jaehun J, Fredenburg JA, Dodani S, Patil PG, Flynn MP. A fully self-contained logarithmic closed-loop deep brain stimulation SoC with wireless telemetry and wireless power management. IEEE J Solid-State Circuits. 2014;49:2213-27.

115. Lin YP, Yeh CY, Huang PY, Wang ZY, Cheng HH, Li YT, et al. A battery-less, implantable Neuro-electronic Interface for studying the mechanisms of deep brain stimulation in rat models. IEEE Trans Biomed Circuits Syst. 2016; 10:98-112.

116. Sommer M, Stiksrud EM, Eckardstein K, Rohde V, Paulus W. When battery exhaustion lets the lame walk: a case report on the importance of long-term stimulator monitoring in deep brain stimulation. BMC Neurol. 2015;15:1-3.

117. Rossi E, Rosa M, Rossi L, Priori A, Marceglia S. WebBioBank: a new platform for integrating clinical forms and shared neurosignal analyses to support multi-centre studies in Parkinson's disease. J Biomed Inform. 2014;52:92-104.

118. Marceglia S, Rossi E, Rosa M, Cogiamanian F, Rossi L, Bertolasi L, et al. Web-based Telemonitoring and delivery of caregiver support for patients with Parkinson disease after deep brain stimulation: protocol. JMIR Res Protoc. 2015;4:e30.

119. C. Yue, H. Hongwei, C. Hao, and L. Luming, "The study on a telemedicine interaction mode for Deep Brain Stimulation postoperative follow-up," 2015 37th Annual International Conference of the IEEE Engineering in Medicine and Biology Society (EMBC), Milan, 2015, pp. 186-189.

120. FDA. (2002, 09 Aug 2017). Medtronic Activa tremor control system P960009. Available at: http://www.accessdata.fda.gov/cdrh_docs/pdf/p960009.pdf.

121. FDA. (2002, 09 Aug 2017). Activa Parkinson's Control System P960009/S7. Available at: https:/www.accessdata.fda.gov/cdrh_docs/pdf/p960009s007b.pdf.

122. FDA. (2003, 09 Aug 2017). Medtronic Activa ${ }^{\circledR}$ Dystonia Therapy - H020007. Available at https://www.accessdata.fda.gov/cdrh_docs/pdf2/H020007A.pdf.

123. FDA. (2009, 09 Aug 2017). FDA Approves Humanitarian Device Exemption for Deep Brain Stimulator for Severe Obsessive-Compulsive Disorder. Available at https:/wayback.archive-it.org/7993/20170113195459/http://www.fda.gov/ NewsEvents/Newsroom/PressAnnouncements/2009/ucm149529.htm. 
124. FDA. (2013, 09 Aug 2017). RNS ${ }^{\oplus}$ System - P100026. Available at https://www. accessdata.fda.gov/cdrh_docs/pdf10/P100026A.pdf.

125. M.-C. Lo and A. S. Widge, "Closed-loop neuromodulation systems: nextgeneration treatments for psychiatric illness," International Review of Psychiatry, 2017;29:191-204.

126. Volkmann J, Moro E, Pahwa R. Basic algorithms for the programming of deep brain stimulation in Parkinson's disease. Mov Disord. 2006;21:S284-9.

127. Ricchi V, Zibetti M, Angrisano S, Merola A, Arduino N, Artusi CA, et al. Transient effects of $80 \mathrm{~Hz}$ stimulation on gait in STN DBS treated PD patients: a 15 months follow-up study. Brain Stimul. 2012;5:388-92.

128. Tass PA. A model of desynchronizing deep brain stimulation with a demand-controlled coordinated reset of neural subpopulations. Biol Cybern. 2003;89:81-8.

129. Tass PA. Desynchronization by means of a coordinated reset of neural subPopulationsA novel technique for demand-controlled deep brain stimulation. Prog Theor Phys Suppl. 2003;150:281-96.

130. M. A. Lourens, B. C. Schwab, J. A. Nirody, H. G. Meijer, and S. A. van Gils, "Exploiting pallidal plasticity for stimulation in Parkinson's disease," J Neural Eng, vol. 12, p. 026005, Apr 2015.

131. O. V. Popovych and P. A. Tass, "Control of Abnormal Synchronization in Neurological Disorders," Frontiers in Neurology, 2014;05:268.

132. P. A. Tass, A. N. Silchenko, C. Hauptmann, U. B. Barnikol, and E. J. Speckmann, "Long-lasting desynchronization in rat hippocampal slice induced by coordinated reset stimulation," Phys Rev E Stat Nonlinear Soft Matter Phys, vol. 80, p. 011902 , Jul 2009.

133. P. A. Tass, L. Qin, C. Hauptmann, S. Dovero, E. Bezard, T. Boraud, et al., "Coordinated reset has sustained aftereffects in Parkinsonian monkeys," Ann Neurol, vol. 72, pp. 816-820, Nov 2012.

134. J. Wang, S. Nebeck, A. Muralidharan, M. D. Johnson, J. L. Vitek, and K. B. Baker, "Coordinated reset deep brain stimulation of subthalamic nucleus produces long-lasting, dose-dependent motor improvements in the 1methyl-4-phenyl-1,2,3,6-tetrahydropyridine non-human primate model of parkinsonism," Brain Stimul, vol. 9, pp. 609-617, 2016/07/01/2016.

135. Adamchic I, Hauptmann C, Barnikol UB, Pawelczyk N, Popovych O, Barniko $\Pi T$, et al. Coordinated reset neuromodulation for Parkinson's disease: proofof-concept study. Mov Disord. 2014:29:1679-84.

136. Tass PA, Adamchic I, Freund HJ, von Stackelberg T, Hauptmann C. Counteracting tinnitus by acoustic coordinated reset neuromodulation. Restor Neurol Neurosci. 2012;30:137-59.

137. A. N. Silchenko, I. Adamchic, C. Hauptmann, and P. A. Tass, "Impact of acoustic coordinated reset neuromodulation on effective connectivity in a neural network of phantom sound," Neurolmage, vol. 77, pp. 133-147, Aug 152013

138. C. Hauptmann, J. C. Roulet, J. J. Niederhauser, W. Doll, M. E. Kirlangic, B. Lysyansky, et al., "External trial deep brain stimulation device for the application of desynchronizing stimulation techniques," J Neural Eng, vol. 6, p. 066003, Dec 2009.

139. Miocinovic S, Somayajula S, Chitnis S, Vitek JL. HIstory, applications, and mechanisms of deep brain stimulation. JAMA Neurology. 2013;70:163-71.

140. Moro E, Poon YY, Lozano AM, Saint-Cyr JA, Lang AE. Subthalamic nucleus stimulation: improvements in outcome with reprogramming. Arch Neurol. Sep 2006;63:1266-72.

141. M. Parastarfeizabadi, A. Z. Kouzani, I. Gibson, and S. J. Tye, "A miniature closed-loop deep brain stimulation device," 2016 38th Annual International Conference of the IEEE Engineering in Medicine and Biology Society (EMBC), Orlando, FL, 2016, pp. 1786-1789.

142. C. A. Gleason, N. F. Kaula, H. Hricak, R. A. Schmidt, and E. A. Tanagho, "The effect of magnetic resonance imagers on implanted neurostimulators," Pacing Clin Electrophysiol, vol. 15, pp. 81-94, Jan 1992.

143. F. G. Shellock, "Magnetic resonance safety update 2002: Implants and devices", J Magn Reson Imaging. 2002;16:485-96.

144. Kuncel AM, Grill WM. Selection of stimulus parameters for deep brain stimulation. Clin Neurophysiol. 2004;115:2431-41.

145. Parastarfeizabadi M, Kouzani AZ. A miniature low-power multi-biomarkerbased brain sensor for closed-loop DBS. IEEE Sensors J. 2017;17:3109-15.

146. A. G. Rouse, S. R. Stanslaski, P. Cong, R. M. Jensen, P. Afshar, D. Ullestad, et al. , "A chronic generalized bi-directional brain-machine interface," J Neural Eng, vol. 8, p. 036018, Jun 2011

147. Eusebio A, Thevathasan W, Doyle Gaynor L, Pogosyan A, Bye E, Foltynie T, et al. Deep brain stimulation can suppress pathological synchronisation in parkinsonian patients. J Neurol Neurosurg Psychiatry. 2011;82:569-73.
148. G. Foffani, G. Ardolino, M. Egidi, E. Caputo, B. Bossi, and A. Priori "Subthalamic oscillatory activities at beta or higher frequency do not change after high-frequency DBS in Parkinson's disease," Brain Res Bull, vol. 69, pp. 123-130, 3/31/ 2006.

149. Jankovic J. Parkinson's disease: clinical features and diagnosis. J Neurol Neurosurg Psychiatry. 2008;79:368-76.

150. Disselhorst-Klug C, Silny J, Rau G. Improvement of spatial resolution in surface-EMG: a theoretical and experimental comparison of different spatial filters. IEEE Transac Biomed Eng. 1997;44:567-74.

151. J. A. Russell and L. Jun, Chapter 4. Nanoelectrode Arrays for Monitoring and Modulating Nervous System Electrical and Chemical Activity, Nanomedicine and the Nervous System, Science Publishers 2012, Pages 60-74.

152. Lee KH, Chang S-Y, Roberts DW, Kim U. Neurotransmitter release from high-frequency stimulation of the subthalamic nucleus. J Neurosurg. 2004:101:511-7.

153. Chang S-Y, Kim I, Marsh MP, Jang DP, Hwang S-C, Van Gompel JJ, et al. Wireless fast-scan cyclic Voltammetry to monitor adenosine in patients with essential tremor during deep brain stimulation. Mayo Clin Proc. 2012;87:760-5.

154. Griessenauer CJ, Chang SY, Tye SJ, Kimble CJ, Bennet KE, Garris PA, et al. Wireless instantaneous neurotransmitter concentration system: electrochemical monitoring of serotonin using fast-scan cyclic voltammetry-a proof-of-principle study. J Neurosurg. 2010;113:656-65.

155. M. Arlotti, L. Rossi, M. Rosa, S. Marceglia, and A. Priori, "An external portable device for adaptive deep brain stimulation (aDBS) clinical research in advanced Parkinson's disease," Med Eng Phys, vol. 38, pp. 498-505, May 2016.

156. Bledsoe JM, Kimble CJ, Covey DP, Blaha CD, Agnesi F, Mohseni P, et al. Development of the wireless instantaneous neurotransmitter concentration system for intraoperative neurochemical monitoring using fast-scan cyclic voltammetry. J Neurosurg. 2009;111:712-23.

157. Abdelhalim K, Jafari HM, Kokarovtseva L, Perez Velazquez JL, Genov R. 64channel UWB wireless neural vector analyzer SOC with a closed-loop phase synchrony-triggered Neurostimulator. IEEE J Solid-State Circuits. 2013:48:2494-510

158. K. Abdelhalim, H. M. Jafari, L. Kokarovtseva, J. L. P. Velazquez, and R. Genov, "64-Channel UWB wireless neural vector analyzer and phase synchronytriggered stimulator SoC," 2012 Proceedings of the ESSCIRC (ESSCIRC), Bordeaux, 2012, pp. 281-284.

159. Bédard C, Kröger H, Destexhe A. Modeling extracellular field potentials and the frequency-filtering properties of extracellular space. Biophys J. 2004:86:1829-42

160. Bédard C, Kröger H, Destexhe A. Model of low-pass filtering of local field potentials in brain tissue. Phys Rev E. 2006;73:051911.

161. Lempka SF, Johnson MD, Moffitt MA, Otto KJ, Kipke DR, McIntyre CC. Theoretical analysis of intracortical microelectrode recordings. J Neural Eng. 2011;:8:045006

162. D. R. Merrill, M. Bikson, and J. G. Jefferys, "Electrical stimulation of excitable tissue: design of efficacious and safe protocols," J Neurosci Methods, vol. 141, pp. 171-198, Feb 152005.

\section{Submit your next manuscript to BioMed Central and we will help you at every step:}

- We accept pre-submission inquiries

- Our selector tool helps you to find the most relevant journal

- We provide round the clock customer support

- Convenient online submission

- Thorough peer review

- Inclusion in PubMed and all major indexing services

- Maximum visibility for your research

Submit your manuscript at www.biomedcentral.com/submit 